\title{
Study protocol: Insight 46 - a neuroscience sub-study of the MRC National Survey of Health and Development
}

Christopher A. Lane ${ }^{1 \dagger}$, Thomas D. Parker ${ }^{1 \dagger}$, Dave M. Cash ${ }^{1,2}$, Kirsty Macpherson $^{1}$, Elizabeth Donnachie $^{3}$, Heidi Murray-Smith ${ }^{1}$, Anna Barnes ${ }^{4}$, Suzie Barker ${ }^{1}$, Daniel G. Beasley ${ }^{2}$, Jose Bras, ${ }^{5,6}$, David Brown ${ }^{4}$, Ninon Burgos ${ }^{2}$, Michelle Byford ${ }^{7}$, M. Jorge Cardoso ${ }^{2}$, Ana Carvalho ${ }^{4}$, Jessica Collins ${ }^{1}$, Enrico De Vita ${ }^{8,9}$, John C. Dickson ${ }^{4}$, Norah Epie', Miklos Espak², Susie M. D. Henley ${ }^{1}$, Chandrashekar Hoskote ${ }^{8}$, Michael Hutel 1,2, Jana Klimova', lan B. Malone ${ }^{1}$, Pawel Markiewicz ${ }^{2}$, Andrew Melbourne ${ }^{2}$, Marc Modat ${ }^{1,2}$, Anette Schrag ${ }^{10}$, Sachit Shah ${ }^{8,9}$, Nikhil Sharma ${ }^{7,11}$, Carole H. Sudre ${ }^{1,2}$, David L. Thomas ${ }^{3,9}$, Andrew Wong ${ }^{7}$, Hui Zhang ${ }^{12}$, John Hardy ${ }^{13}$, Henrik Zetterberg ${ }^{5,14,15}$, Sebastien Ourselin², Sebastian J. Crutch ${ }^{1}$, Diana Kuh', Marcus Richards ${ }^{7}$, Nick C. Fox ${ }^{1}$ and Jonathan M. Schott ${ }^{*}$

\begin{abstract}
Background: Increasing age is the biggest risk factor for dementia, of which Alzheimer's disease is the commonest cause. The pathological changes underpinning Alzheimer's disease are thought to develop at least a decade prior to the onset of symptoms. Molecular positron emission tomography and multi-modal magnetic resonance imaging allow key pathological processes underpinning cognitive impairment - including $\beta$-amyloid depostion, vascular disease, network breakdown and atrophy - to be assessed repeatedly and non-invasively. This enables potential determinants of dementia to be delineated earlier, and therefore opens a pre-symptomatic window where intervention may prevent the onset of cognitive symptoms.

Methods/design: This paper outlines the clinical, cognitive and imaging protocol of "Insight 46", a neuroscience sub-study of the MRC National Survey of Health and Development. This is one of the oldest British birth cohort studies and has followed 5362 individuals since their birth in England, Scotland and Wales during one week in March 1946. These individuals have been tracked in 24 waves of data collection incorporating a wide range of health and functional measures, including repeat measures of cognitive function. Now aged 71 years, a small fraction have overt dementia, but estimates suggest that $\sim 1 / 3$ of individuals in this age group may be in the preclinical stages of Alzheimer's disease. Insight 46 is recruiting 500 study members selected at random from those who attended a clinical visit at 60-64 years and on whom relevant lifecourse data are available. We describe the sub-study design and protocol which involves a prospective two time-point (0, 24 month) data collection covering clinical, neuropsychological, $\beta$-amyloid positron emission tomography and magnetic resonance imaging, biomarker and genetic information. Data collection started in 2015 (age 69) and aims to be completed in 2019 (age 73).

(Continued on next page)
\end{abstract}

\footnotetext{
* Correspondence: j.schott@ucl.ac.uk

${ }^{\dagger}$ Equal contributors

'Dementia Research Centre, Institute of Neurology, University College

London, London, UK

Full list of author information is available at the end of the article
} International License (http://creativecommons.org/licenses/by/4.0/), which permits unrestricted use, distribution, and reproduction in any medium, provided you give appropriate credit to the original author(s) and the source, provide a link to the Creative Commons license, and indicate if changes were made. The Creative Commons Public Domain Dedication waiver (http://creativecommons.org/publicdomain/zero/1.0/) applies to the data made available in this article, unless otherwise stated. 
(Continued from previous page)

Discussion: Through the integration of data on the socioeconomic environment and on physical, psychological and cognitive function from 0 to 69 years, coupled with genetics, structural and molecular imaging, and intensive cognitive and neurological phenotyping, Insight 46 aims to identify lifetime factors which influence brain health and cognitive ageing, with particular focus on Alzheimer's disease and cerebrovascular disease. This will provide an evidence base for the rational design of disease-modifying trials.

Keywords: Epidemiology, Life course, Genetics, Alzheimer's Disease, Ageing, Magnetic resonance imaging, Positron emission tomography, Cognition, Vascular disease, Birth cohort

\section{Background}

Dementia is the leading cause of death in England and Wales, accounting for $11.6 \%$ of all deaths registered in 2015 [1]. As the population ages, the burden of neurological diseases and dementia in particular will increase dramatically. Current estimates suggest that 44 million people worldwide are currently living with dementia, and this number is predicted to more than triple by 2050, by which time the annual cost of dementia in the US alone may exceed US\$604 billion [2]. Estimates suggest that a five-year delay in symptom onset would halve prevalence, costs and burden [3]. Understanding the causes of dementia, and lifestyle or pharmacological interventions that can prevent or delay the onset of symptoms is therefore a global priority.

Dementia is a clinical syndrome due to many underlying diseases, of which Alzheimer's disease (AD) is the commonest single cause. AD is characterised histopathologically by the accumulation of senile plaques mainly composed of amyloid $\beta(\mathrm{A} \beta)$, neurofibrillary tangles composed of hyperphosphorylated tau [4], and excess neuronal cell loss (atrophy) in vulnerable regions, notably the medial temporal lobe and parietal association cortices. The emergence of techniques for studying biomarkers allows for many aspects of $\mathrm{AD}$ pathology to be assessed in vivo. In particular, positron emission tomography (PET) using amyloid-specific tracers allows for quantification of fibrillar amyloid burden; and modern multi-modal magnetic resonance imaging (MRI) offers a non-invasive way of determining brain volumes, cerebrovascular disease, white matter tract integrity, brain perfusion, functional connectivity, and brain microstructure. Applying many of these techniques to cohorts with rare, autosomal dominantly inherited forms of $\mathrm{AD}$, sporadic $\mathrm{AD}$, and healthy older controls suggests that: (1) accumulation of $A \beta$ is seen in a significant proportion (up to a third) of individuals in their 70s; (2) $\mathrm{A} \beta$ accumulation occurs prior to, and is likely to trigger, the development of other pathological processes core to $\mathrm{AD}$, including the deposition and spread of abnormally hyperphosphorlayed tau through vulnerable networks, microglial activation, brain hypometabolism, and increased rates of atrophy; and (3) these processes all occur several years - and in the case of amyloid deposition perhaps a decade or more - prior to the development of symptoms $[5,6]$.

These findings have already led to important advances, including (1) a re-conceptualisation of $\mathrm{AD}$ to include healthy individuals at risk, with contemporary research criteria now determining that asymptomatic individuals with evidence for brain amyloid, brain amyloid + neurodegeneration, or brain amyloid + neurodegeneration + subtle cognitive impairment can be designated as having preclinical AD [7-9]; and (2) the advent of clinical trials in asymptomatic participants either at risk of $\mathrm{AD}$ on the basis of carrying a gene known to cause familial $\mathrm{AD}[10,11]$ or individuals with asymptomatic amyloidosis [12]. These trials aim to modify aspects of AD pathology by slowing or even reversing the development of brain pathology, and delaying the onset of cognitive decline and ultimately the clinical manifestation of AD dementia.

Our understanding of this presymptomatic period is, however, far from complete. Evidence to date comes largely from extrapolation of cross-sectional (or short-interval longitudinal follow-up) studies to infer the sequence of changes that occur over much longer periods [13]. Individuals selected for such studies often are not typical of the population as a whole, with many having genetic risks factors, concerns about cognition, or a family history of dementia [14]. Little is known in the general population about the factors that influence the development, sequence and timing of the different pathologies implicated in $\mathrm{AD}$, and how they interact with other brain pathologies (e.g. cerebrovascular disease) to influence cognitive function. Consequently, the evidence base to inform the design of clinical trials in the presymptomatic phase is currently limited.

In parallel with the need to identify preclinical $\mathrm{AD}$ for interventional trials, it is also vital to understand what influences an individual's risk of developing $\mathrm{AD}$ and other forms of late-life cognitive impairment. There are more than 20 identified genetic risk factors for AD, most of which exert only a small influence on risk, but together, by way of a polygenic risk score, have been shown to almost double case prediction from chance [15]. There is evidence that education and physical exercise are protective, whereas mid-life hypertension, obesity and diabetes 
adversely influence risk [16]. However it is unclear whether these factors act independently, cumulatively or interactively, and how they influence different pathological processes that can lead to dementia; to address these questions requires prospective data across the life course.

The Medical Research Council (MRC) National Survey of Health and Development (NSHD) has followed 5362 individuals since their birth in England, Scotland and Wales during one week in March 1946 [17-19]. Repeated waves of data collection since childhood have enabled detailed cognitive and physical phenotyping of this population-representative cohort. Details of the cohort are provided elsewhere $[17,18]$, with an overview of relevant information collected to date in Table 1. Now aged 71, members of this intensively-studied cohort are at a critical age to investigate preclinical AD: old enough to be at high risk for amyloid pathology, but several years before the expected exponential rise in dementia prevalence [20].

We describe here the study design and protocol of "Insight 46", a prospective longitudinal two time-point (0, 24 month) sub-study of 500 study members, incorporating the collection of new clinical, neuropsychological, MRI, PET amyloid imaging, and blood and urine biomarkers. We outline the study's organisation and funding structure, provide an overview of the recruitment criteria, the cognitive, imaging and fluid biomarker protocols, and the duty of care protocol. We summarise the key hypotheses to be tested, and the data that are being collected; these data will in due course be made available to the research community.

\section{Methods/design \\ Study organisation/funding}

Insight 46 is funded by grants from Alzheimer's Research UK (ARUK-PG2014-1946, ARUK-PG2017-1946 PIs Schott, Fox, Richards), the Medical Research Council Dementias Platform UK (CSUB19166 PIs Schott, Fox, Richards), the Wolfson Foundation (PR/ylr/18575 PIs Fox, Schott), the Medical Research Council (MC_UU_12019/1 PI Kuh and MC_UU_12019/3 PI Richards), the Wellcome Trust (Clinical Research Fellowship 200109/Z/15/Z Parker) and Brain Research Trust (UCC14191, PI Schott). AVID Radiopharmaceuticals (a wholly owned subsidiary of Eli Lilly) provide the PET amyloid tracer (Florbetapir) but had no part in the design of the study.

Separate ethical approvals for NSHD have been provided by Research Ethics Committees in England and Scotland outlined elsewhere [17, 19, 21]. Ethical approval for the neuroscience sub-study was granted by the National Research Ethics Service (NRES) Committee London (REC reference 14/LO/1173, PI Schott). All participants provide written informed consent to participate

Table 1 Overview of life course data available for MRC NSHD study participants

\begin{tabular}{|c|c|c|c|c|c|c|c|c|c|}
\hline & & & \multicolumn{7}{|c|}{ Time point (ages) } \\
\hline & & & $\begin{array}{l}1946 \\
\text { (birth) }\end{array}$ & $\begin{array}{l}1947-50 \\
(1-4 \text { yrs })\end{array}$ & $\begin{array}{l}1951-61 \\
(5-15 y r s)\end{array}$ & $\begin{array}{l}1962-77 \\
(16-31 \text { yrs })\end{array}$ & $\begin{array}{l}1978-2003 \\
(32-57 \text { yrs })\end{array}$ & $\begin{array}{l}2006-10 \\
(60-64 \text { yrs })\end{array}$ & $\begin{array}{l}2014-15 \\
(68-69 \text { yrs })\end{array}$ \\
\hline \multicolumn{3}{|c|}{ Number of data collections } & 1 & 2 & 8 & 8 & 3 & 1 & 1 \\
\hline \multirow[t]{16}{*}{ Measure } & \multirow[t]{4}{*}{ Social factors } & Socioeconomic position & $\checkmark$ & $\checkmark$ & $\checkmark$ & $\checkmark$ & $\checkmark$ & $\checkmark$ & $\checkmark$ \\
\hline & & $\begin{array}{l}\text { Social function (contacts, support, } \\
\text { participation) }\end{array}$ & - & - & - & - & $\checkmark$ & $\checkmark$ & $\checkmark$ \\
\hline & & Occupation & - & - & - & $\checkmark$ & $\checkmark$ & $\checkmark$ & $\checkmark$ \\
\hline & & Educational Qualifications & & & $\checkmark$ & $\checkmark$ & & & \\
\hline & $\begin{array}{l}\text { Psychological } \\
\text { measures }\end{array}$ & Behaviour and mental health & - & $\checkmark$ & $\checkmark$ & $\checkmark$ & $\checkmark$ & $\checkmark$ & $\checkmark$ \\
\hline & \multirow{10}{*}{$\begin{array}{l}\text { Physical and health } \\
\text { measures }\end{array}$} & Survival and morbidity & $\checkmark$ & $\checkmark$ & $\checkmark$ & $\checkmark$ & $\checkmark$ & $\checkmark$ & $\checkmark$ \\
\hline & & Anthropometric measures & $\checkmark$ & $\checkmark$ & $\checkmark$ & $\checkmark$ & $\checkmark$ & $\checkmark$ & $\checkmark$ \\
\hline & & Smoking status & - & - & - & $\checkmark$ & $\checkmark$ & $\checkmark$ & $\checkmark$ \\
\hline & & Exercise and physical health & - & - & $\checkmark$ & - & $\checkmark$ & $\checkmark$ & $\checkmark$ \\
\hline & & Diet & - & $\checkmark$ & - & - & $\checkmark$ & $\checkmark$ & $\checkmark$ \\
\hline & & Respiratory function & - & - & - & - & $\sqrt{ }(36,43,53)$ & $\checkmark$ & $\checkmark$ \\
\hline & & Cardiovascular function & - & - & - & - & $\sqrt{ }(36,43,53)$ & $\checkmark$ & $\checkmark$ \\
\hline & & Musculoskeletal measures & - & - & - & - & $\checkmark(53)$ & $\checkmark$ & $\checkmark$ \\
\hline & & Blood sample & - & - & - & - & $\checkmark(53)$ & $\checkmark$ & $\checkmark$ \\
\hline & & Urine sample & - & - & - & - & - & $\checkmark$ & - \\
\hline & Cognition & $\begin{array}{l}\text { Cognitive function } \\
\text { (verbal/non-verbal) }\end{array}$ & - & - & $\checkmark(8,11,15)$ & $\checkmark(26)$ & $\checkmark(43,53)$ & $\checkmark$ & $\checkmark$ \\
\hline
\end{tabular}


and for their data to be stored in accordance with the Data Protection Act.

\section{Participants' recruitment and clinical protocol description Participants}

To capitalise on the life course data and to avoid a priori decisions as to who might be at risk of cognitive decline, entry criteria to the sub-study are based only on maximising the life course data available for analysis. A sample of 500 NSHD study members are being selected at random from those who attended a clinic-based assessment age 60-64, had previously intimated they were willing to attend a clinic visit in London and for whom relevant data in childhood and adulthood are available. These relevant data are shown in Table 2.

The first 500 study members fulfilling these criteria and agreeing to participate will be included. Excluded are individuals with contraindications to MRI or PET including, but not limited to, claustrophobia, metallic implants such as pacemakers, or research nuclear medicine scans within the last year that would result in an individual exceeding acceptable mandated yearly radiation exposures. Where appropriate, the option to consent to post-mortem brain donation is discussed with participants. A flowchart outlining the study is shown in Fig. 1.

\section{Duty of care}

A duty of care protocol building on the NSHD protocol used in 2006-10 and in accordance with the MRC/Wellcome Trust guidelines is being implemented for the purpose of feeding back health-related findings in research [22] to each participant and their GP. Participants are given the option of 'opting out'

Table 2 Minimum life course dataset for Insight 46

Attendance at a clinic visit at age 60-64

Parental socioeconomic position: at least one indicator of occupational social class or education

Cognition: memory and processing speed from the 60-64 year collection AND at least one set of measures at either ages 8,11 or 15

Early physical growth trajectories: birth weight and at least one measure of height and weight at ages 4-15

Educational attainment: highest qualification by age 26

Mental health: teacher ratings of behaviour and temperament at ages 13 or 15 , and at least one measure of affective symptoms at ages $36,43,53$ or $60-64$

Blood pressure, lung function, adult height and weight: at least one measure of each at ages $36,43,53$ or $60-64$

Health behaviours: at least one measure of smoking and physical exercise at ages $36,43,53$ or $60-64$

Blood: either age 53 or 60-64 samples from receiving any correspondence regarding reportable findings, but must consent to their GP receiving the information. Anthropomorphic measures (height and weight), recumbent blood pressure, audiometry and a range of standard clinical blood tests (haemoglobin, platelet count, vitamin B12, urea, creatinine, random glucose and TSH) together with their normal ranges are routinely reported. Participants with results outside the normal range are advised to discuss the results with their GP in a timely fashion. If blood results are significantly outside the normal range, falling beyond pre-specified 'Action' levels, the study clinician contacts the participant and GP via telephone within $48 \mathrm{~h}$ of receipt of results.

All T1, T2 and FLAIR volumetric MRI sequences are reviewed by a consultant neuroradiologist at the National Hospital for Neurology and Neurosurgery. The study follows guidelines based on the UK Biobank imaging study (www.ukbiobank.ac.uk/wp-content/uploads/2016/11/ Incidental-findings-list-of-possible-abnormalities.pdf), and information is only fed back to study members and their GP if there is an MRI abnormality that might require treatment or surveillance. A list of potential findings considered reportable are summarised in Table 3. The ethical challenges of providing information regarding amyloid PET results in cognitively-normal individuals has been discussed elsewhere [23], and for this study amyloid PET status is not fed back to study members.

In addition, information is fed back to participants and their GP if clinical assessments reveal clear evidence of significant cognitive impairment (based on a MMSE score $\leq 24$ and/or significant concern from study clinician) or clinically detectable parkinsonism (i.e. fulfilling Queen Square Brain Bank criteria [24] for Parkinson's disease (PD)) in previously undiagnosed individuals. Appendix 1 summarises reportable findings and normal ranges employed in Insight 46.

Clinical, neurological, cognitive and sensory assessments All individuals are assessed at a single site (UCL). Participants complete four self-administered questionnaires; undergo a structured clinical interview with a neurologist; have a structured neurological examination; undergo neuropsychological testing, and assessment and testing of auditory, olfactory and visual function. These assessments have been designed to be administered in divided sessions that last less than four hours during a single day (mean duration $=199$ min based on six pilot visits).

\section{Self-administered questionnaires}

The state and trait anxiety inventory [25]: This 40-item questionnaire assesses an individual's thoughts and feelings, and is designed to quantify anxiety levels at the present moment and in general. 


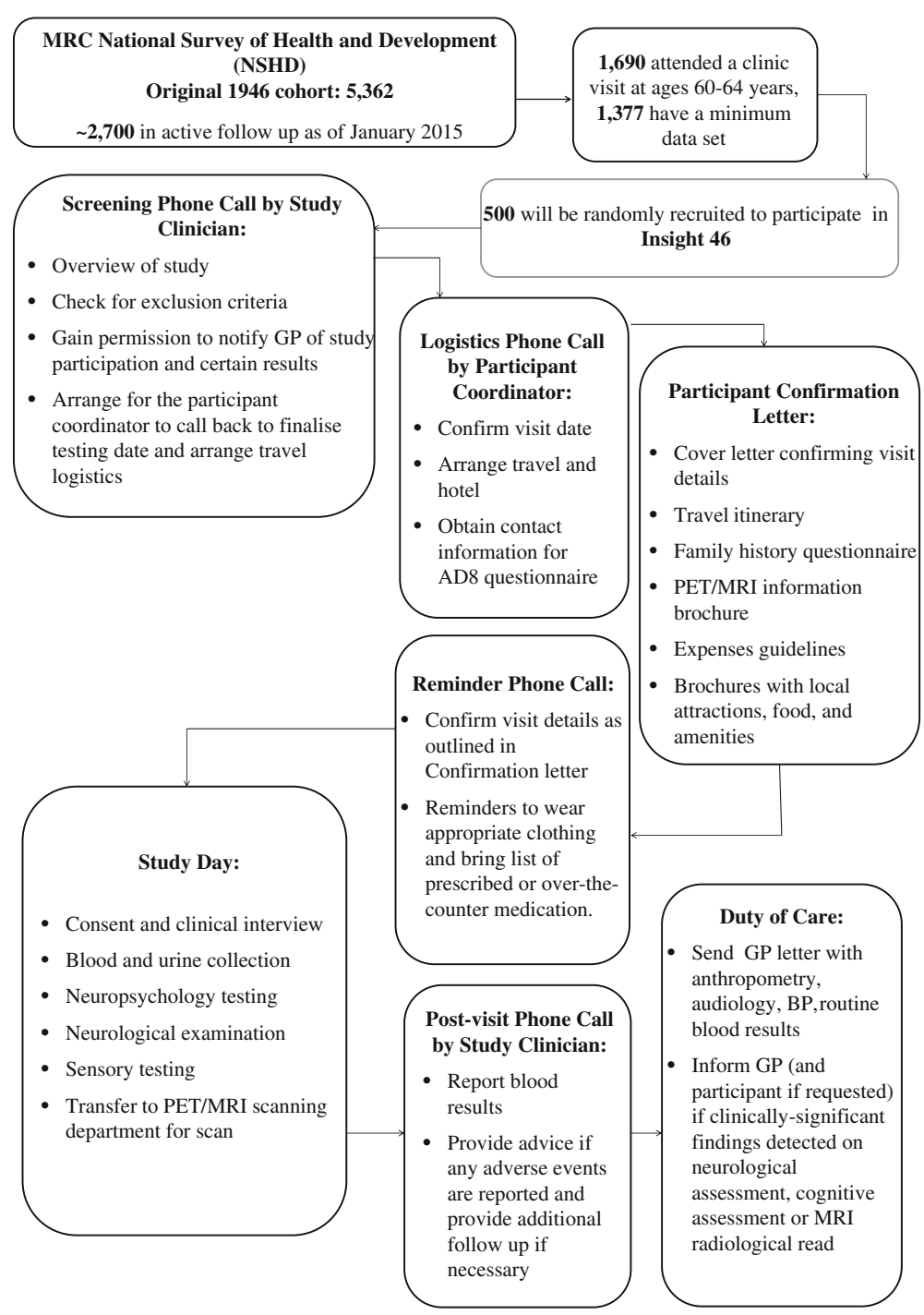

Fig. 1 Flowchart for Insight 46

Table 3 Reportable MRI brain findings

\section{Acute brain infarction \\ Acute brain haemorrhage (note: not old bleeds)}

Intracranial mass lesions (note: not meningiomas in locations considered highly unlikely to cause problems)

Suspected intracranial aneurysm or vascular malformation (inc. cavernomata) (note: not aneurysms less than $7 \mathrm{~mm}$ in diameter)

\section{Colloid cyst of the 3rd ventricle}

Acute hydrocephalus

Significant sinus disease with suspicion of underlying pathology (e.g. unilateral sinus opacification)

Other unexpected, serious, or life-threatening findings
A dental health questionnaire [26]: There is growing evidence that periodontitis is a risk factor for sporadic $\mathrm{AD}$ and it has been postulated that periodontal pathogens may drive chronic neuro-inflammation contributing to Alzheimer's pathology [27]. This self-administered questionnaire utilises eight questions designed to assess the likelihood of periodontitis.

$A$ handedness questionnaire [28]: Hand preference is closely related to cerebral dominance [29]. This questionnaire asseses hand preference for 12 different tasks using a numerical scale enabling quantification of each participant's handedness (range -24 to +24 ).

Screening question for Rapid Eye Movement (REM) sleep behaviour disorder [30]: REM sleep behaviour disorder is a known risk factor for the emergence of 
Parkinson's disease and related disorders. A yes/no answer is obtained to the question "Have you ever been told, or suspected yourself, that you seem to 'act out your dreams' while asleep (for example, punching, flailing your arms in the air, making running movements, etc.)?". This has a sensitivity of $93.8 \%$ and a specificity of $87.2 \%$ for detecting REM sleep behaviour disorder [30].

\section{Clinical interview}

A standard personal and family history of neurological illness or cognitive impairment and a medication history is obtained. In addition, participants are screened for measures of self-perceived cognitive decline using the SCD-Q part I (MyCog) questionnaire [31] and are asked questions that enable coding of essential features of subjective cognitive decline as outlined by the working group of the Subjective Cognitive Decline Initiative [32]. A corroborative history regarding each participant's cognitive functioning is obtained using the AD8 screening tool, an informant questionnaire administered in person or via the telephone by the study clinician. The AD8 correlates well with the clinical dementia rating scale (CDR), and has high sensitivity and specificity for detecting cognitive impairment [33, 34].

\section{Physical and neurological examination}

A physical examination comprises anthropomorphic measures (weight in kilograms and height measured to the nearest $\mathrm{mm}$ ), and lying and standing blood pressure at three minutes to assess for evidence of orthostatic hypotension (OMRON HEM-905; OMRON Healthcare UK Ltd., Milton Keynes).

Patients with $\mathrm{AD}$ and other forms of dementia have more marked decline in motor function, including gait, than healthy controls, with the possibility that these changes may precede the onset of frank cognitive symptoms $[35,36]$. This is perhaps not surprising if gait is viewed as a complex cognitive task, requiring an interplay of attention, executive function and visuospatial function, in addition to the motor processing functions of the motor cortex, basal ganglia and cerebellum. It has therefore been suggested that changes in gait and motor skills in general may reflect and correlate with early cognitive change [37].

Participants' self-paced gait is assessed over a 20$\mathrm{m}$ distance in isolation and while performing a cognitive task (single-letter-cued (phonemic) fluency and dual-letter-cued (phonemic) alternate fluency), and wearing an accelerometer on the lower back (LPMS-B inertial measurement unit (Life performance Research Inc)), with data analysis using a custom program written in LabVIEW2010 (National
Instruments, Ireland). Temporal (step time and cadence) as well as spatial (step-, stride-time, walking speed) parameters can be derived. More indepth analysis utilising temporal-spatial parameters and participant metadata can describe parameters indicating motor control [38]. Non-linear or phase plot analysis can be used to explore subtle gait changes using the whole dataset of a particular participant. Using this approach, it has been demonstrated that changes in gait can be detected in the pre-symptomatic phase of Huntington's disease [39].

Although parkinsonian features are generally considered to be later clinical manifestations in $\mathrm{AD}$, it has been reported that parkinsonian features may precede the onset of frank dementia [40, 41]. A standardised neurological examination includes the MDS-Unified Parkinson's Disease Rating Scale (UPDRS) Part III (Motor) [42], which quantifies presence of tremor, bradykinesia, rigidity, postural instabilty and gait disorder. Assessments are videotaped for quality control purposes and to enable futher review by a senior neurologist if clinicallysignificant parkinsonian features are identified. The Bradykinesia Akinesia Incoordination (BRAIN) test is administered to all participants via a laptop (Lenovo Thinkpad, Lenovo Group Ltd). This computer keyboardtapping task was originally developed for use in assessing the effect of symptomatic treatment on motor function in Parkinson's disease. An online version has been designed and validated which can be utilised as an objective longitudinal measure of emerging motor dysfunction [43]. The outputs of the BRAIN test include a kinesia score (KS30, number of key taps in $30 \mathrm{~s}$ ), akinesia time (AT30, mean dwell time on each key in $\mathrm{ms}$ ), incoordination score (IS30, variance of travelling time between key presses) and dysmetria score (DS30, accuracy of key presses).

\section{Cognitive battery}

The cognitive assessment battery is based on a review of results and cognitive protocols from several large-scale initiatives and clinical trials involving individuals at risk for $\mathrm{AD}[10,12,44]$, and is complementary to cognitive assessments performed as part of the most recent (in some cases concurrent) NSHD home visit (that includes the ACE-III [45] and word-list learning [46]). Complementing ongoing work with the whole NSHD cohort, participants' cognitive trajectories will be assessed prospectively over the two time points, and retrospectively using previously collected cognitive measures (refer to Appendix 2 for an overview of neuropsychometric tests collected to date). 
The battery includes:

The mini-mental state examination (MMSE) [47]

The MMSE is a widely used 30-point screening tool for cognitive impairment within clinical practice, assessing multiple cognitive domains including: $i$ ) orientation to time and place (10 points); ii) registration (3 points); iii) attention $+/$ - calculation (5 points); $i v$ ) recall (3 points); $v$ ) language (2 points); vi) repetition (1 point); vii) reading (1 point); viii) writing (1 point); $i x$ ) visuospatial function (1 point); $x$ ) following a 3 -stage command (3 points).

Logical memory from the Wechsler Memory Scale-Revised (WMS-R) [48]

The Logical Memory test assesses free recall of a short story that contains 25 details. The participant is asked to recall the story immediately and after a delay of approximately $20 \mathrm{~min}$.

Digit-symbol substitution test, from the Wechsler Adult Intelligence Scale-Revised (WAIS-R) [49]

The Digit-Symbol Substitution test explores attention and psychomotor speed. Participants are given a code table displaying the digits from 1 to 9 , each paired with a symbol. On a worksheet printed with rows of digits, participants are asked to fill in the corresponding symbol under each digit as shown in the code table, as quickly and accurately as possible. The score is the number of symbols completed correctly within $90 \mathrm{~s}$.

Matrix reasoning from the Wechsler Abbreviated Scale of Intelligence (WASI) [50]

The Matrix Reasoning test assesses non-verbal reasoning. Participants are shown a matrix of geometric shapes with a section missing and are required to select the missing piece from five options. There are 32 matrices, graded in difficulty, and the test is discontinued when participants reach a certain error threshold, as specified in the WASI manual.

Five more novel tests, intended to detect subtle, early cognitive deficits, are also being administered (see Fig. 2).

Task-set switching / response inhibition [51, 52]

A meta-analysis of relationships between amyloid burden and cognition in cognitively-normal older adults found evidence of an association between amyloid burden and executive functioning [53]. This task examines the relationship between two important executive functions - task-set switching and response inhibition which are vulnerable in early $\mathrm{AD}[54,55]$. Individuals are presented with a computer screen on which a stimulus is displayed, and a response box with two buttons. The first part of the experiment comprises the simple choice "arrow only" and "word only" conditions, which complements the simple choice reaction time tasks administered at 60-64 years. In the "arrow only" condition, participants are shown the cue 'arrow' for $1000 \mathrm{~ms}$, followed by an arrow pointing left or right. In the "word only" condition, participants are shown the cue 'word' for $1000 \mathrm{~ms}$, followed by the word 'left' or 'right'. In each case they must press the button that corresponds to the stimulus, using the index and middle fingers of their dominant hand. The second part of the experiment is a switching condition in which the cue may be either 'arrow' or 'word', and the stimulus is a combined arrow and word. The stimulus is either congruent (e.g. left arrow and the word 'left') or incongruent (e.g. left arrow and and the word 'right'). Trials in the switching task are categorised into switch and non-switch. In a non-switch trial the cue is the same as for the immediately preceding trial, whereas in a switch trial the cue differs from the immediately preceding trial. In order to evaluate the effect of preparation time on task-set switching and response inhibition, the cue is shown for either a short (200 ms) or long (1500 ms) interval before the stimulus appears. Outcome measures are reaction time and error rate.

'What was where?' visual short-term memory binding task $[56,57]$

This test requires participants to view one or three fractal objects, presented simultaneously in random locations on the screen. Participants are asked to remember both the objects and their locations. After a delay of one or four seconds they are required to make a forced choice between two fractals, one of which was displayed in the initial memory array (the target) and the other of which is a 'dummy' fractal. Participants are required to touch the object they think has been previously presented and 'drag' it on the touch screen to its remembered, original location. Outcome measures are the proportion of fractals correctly identified, and the localisation error (i.e. the distance between the location reported by the participant and the true location of the target in the initial memory array) and the proportion of 'binding errors'. A binding error occurs when a participant chooses the correct fractal but drags it to the location of one of the non-target (unprobed) fractals from the initial array. The binding of such featural information has been shown to be vulnerable in asymptomatic familial AD mutation carriers $[58,59]$. There is evidence that binding ability is relatively preserved in normal ageing despite the age-related decline in memory for object identification and localisation, making it a promising target for sensitive tests to detect preclinical $\mathrm{AD}[60]$.

\section{Visuomotor integration}

This is a circle-tracing task which includes both direct and indirect visual feedback conditions. The task is presented on a tablet laptop, with the screen placed flat on the table in front of the participant, with an additional free-standing monitor behind it. Participants are asked to use a stylus to trace round a circle on the tablet as quickly and accurately as possible. In the direct 

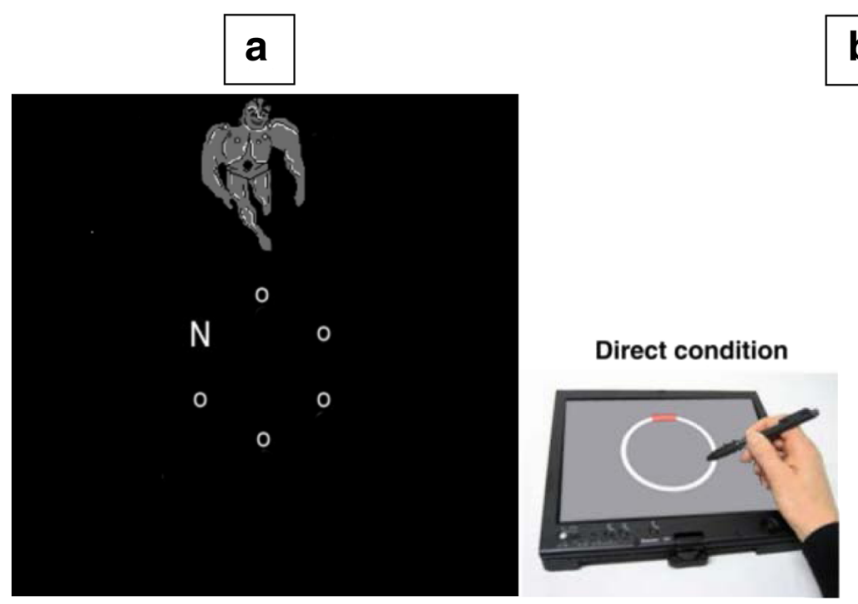

b
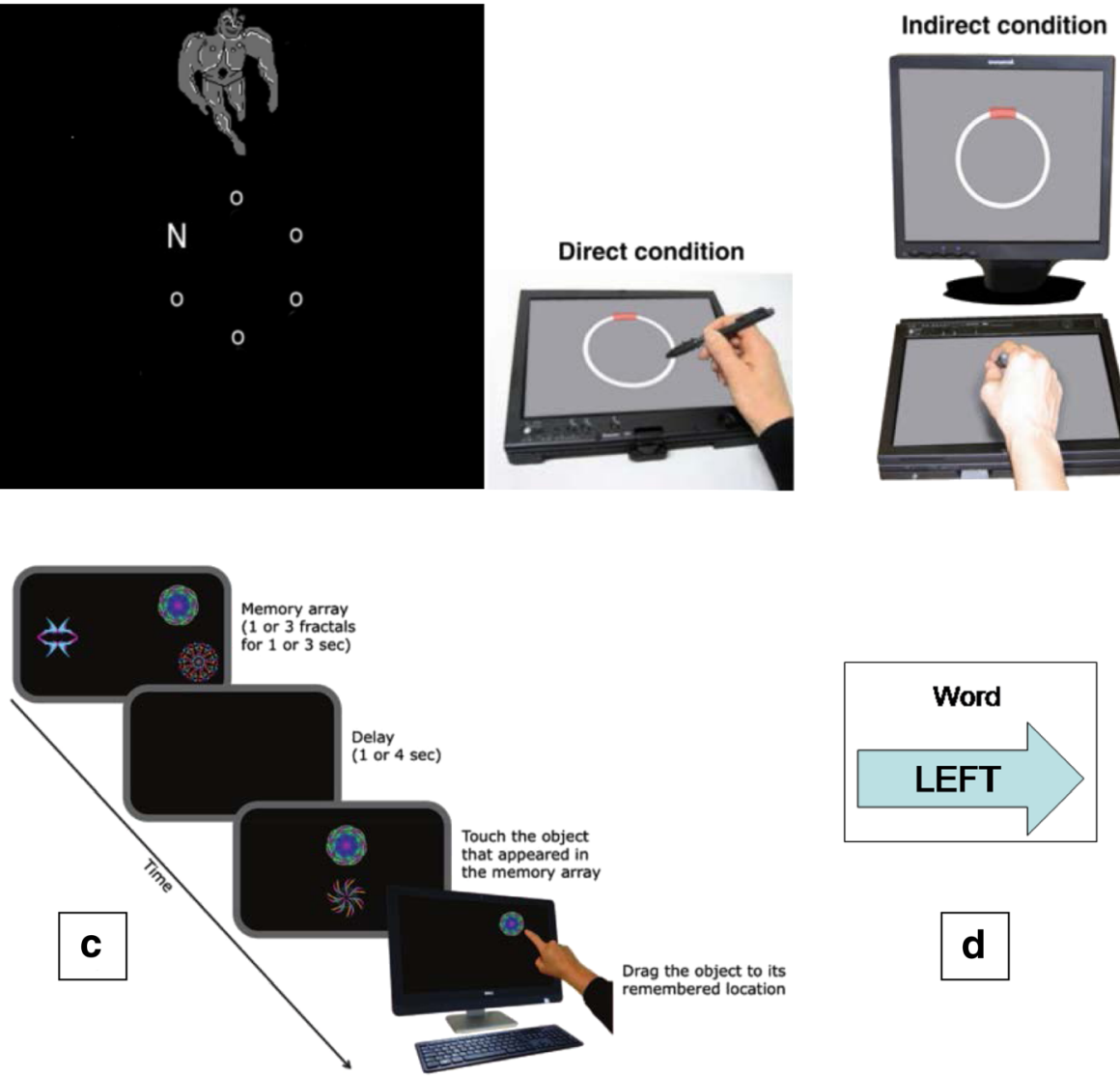

Fig. 2 Novel computerised tests (a) Irrelevant Distractor. An example stimulus display (not to scale) with an irrelevant distractor in the low load condition. Note that the specific cartoon image shown here as an irrelevant distractor is included for illustrative purposes only, in order to avoid violating copyright for the images used in the experiment. Figure reprinted from [64] with permission from American Psychological Association (b) Visuomotor Integration apparatus. Note that in the indirect condition, the participant's hand is covered by a box, not shown here. Figure reprinted from [61], Copyright, with permission from Elsevier. (c) 'What was where?' task. Figure reprinted from [56] available from https://www.ncbi.nlm.nih.gov/pmc/articles/PMC4360752/, American Psychological Association, copyright under the Creative Commons Attribution License https://creativecommons.org/licenses/by/3.0/ (d) Task-set Switching / Response Inhibition. An example stimulus display for an incongruent word trial

condition, participants can see their hand and the path they are tracing on the tablet. In the indirect condition, participants put their arm under a box so they cannot see their hand, but they are instructed to look at the free-standing monitor to view a copy of the circle and their tracing path. Continuous performance measures are provided including accuracy, speed and speed of error detection and correction. The test has revealed changes in speed and accuracy in Huntington's Disease mutation carriers more than 10 years before expected age-of-onset [61].

12-item Face-Name Associative Memory Exam (FNAME12A)

The FNAME-12A is a modified version of the 16-item Face-Name Associative Memory Exam (FNAME-16). The FNAME-12A has fewer stimuli and additional learning trials which are well tolerated by those with mild cognitive impairment (MCI), while remaining challenging in cognitively-normal older adults [62]. It has demonstrated psychometric equivalence with the FNAME-16, which is related to $\beta$-amyloid burden in cognitivelynormal older people [63]. The FNAME-12A requires the participant to learn 12 face-name and face-occupation pairs. Participants are given two exposures to all 12 facename/occupation pairs. After each exposure and following a 10-min delay they are asked for the name and occupation associated with each face. After a 35-min delay they are shown three faces and asked to identify each previously learned face from two distractors (facial recognition) and to recall the name and occupation. If they cannot remember the name or occupation, they are provided with three recognition choices. 


\section{Irrelevant distractor paradigm $[64,65]$}

Participants are given a computerised letter-search task and are required to make a rapid decision as to which target letter (' $\mathrm{X}$ ' or ' $\mathrm{N}$ ') has appeared in the search display. There are three load conditions, high (four letters), medium (three letters) and low (one letter). On some of the trials, a distractor appears on the outside of the search display. This distractor can either be taskirrelevant (a cartoon character) or task-relevant (the letter $\mathrm{X}$ or $\mathrm{N}$ ). The task-relevant distractors can be congruent or incongruent to the target letter. Outcome measures are reaction time and error rate. The task evaluates the extent to which attention is captured by the different distractors, and the role of perceptual load in this process.

These tasks give weight both to response accuracy and latency to maximize detection of subtle cognitive change and discriminate cortical/subcortical dysfunction. Cognitive performance at the 2-year follow up in the sub-study will be evaluated against study baseline performance and cognitive performance in childhood, adolescence and adulthood.

\section{Sensory function}

There is increasing interest in the possibility that impaired visual function, sense of smell and hearing may provide signals of preclinical AD [66-70]. As such, participants will have comprehensive sensory assessments of vision, olfaction and both peripheral and central auditory function.

Basic parameters of visual function, an important factor contributing to deficits experienced in elderly patients with cognitive impairment, are assessed using The Portable Eye Examination Kit (PEEK), a smartphone application that measures visual acuity, colour vision and contrast sensitivity [71].

The University of Pennsylvania Smell Identification Test (UPSIT) is a commercially-available, well-established, reliable, and standardized olfactory test that can be selfadministered [72]. The 'British' version is being used as it is the most culturally appropriate to the NSHD. Each test comprises four 10-page booklets with one odorant (embedded in 10-50- $\mu \mathrm{m}$ diameter microcapsules fixed in a proprietary binder and positioned on brown strips) at the bottom of each page. Accompanying each strip is a multiple-choice question with four responses following an alternative forced-choice paradigm. Packs are provided to participants to complete at home and returned using a pre-paid envelope provided. Participants are also asked prior to testing whether they have subjectively noticed a decline in their sense of smell.

Peripheral hearing is assessed using air conduction threshold audiometry, with pure tones presented to each ear at different frequencies covering the range of human speech perception $(0.5 \mathrm{kHz}-4 \mathrm{kHz})$. A testing procedure in keeping with British Society of Audiology recommendations [73] is used in which the sound level of the tones are varied and enable hearing thresholds for each ear at each frequency to be obtained.

Central auditory processing is tested by using a word identification in background noise paradigm [74]. This involves presentation of high-frequency monosyllabic words embedded in a multi-talker babble noise composed of 20 voices. The background noise is presented at a fixed level of $65 \mathrm{~dB}$ SPL, while the sound level of each individual word is varied according to an adaptive staircase procedure based on whether participants are able to identify the word correctly. This is designed to obtain a "speech reception threshold", which quantifies participants' ability to identify spoken words in background noise.

\section{Imaging protocol description}

Imaging is performed on a Biograph mMR $3 \mathrm{~T} \mathrm{PET/}$ MRI scanner (Siemens Healthcare, Erlangen), allowing for simultaneous acquisition of dynamic amyloid PET and MR data whilst minimising scanning time and exposure to radiation (compared with the use of PETCT). Participants will have one scanning session at each time point. The neuroimaging protocol comprises both structural and functional acquisitions, and is designed to be completed within a $60-\mathrm{min}$ scanning session.

Amyloid load is assessed using the ${ }^{18} \mathrm{~F}$ amyloid PET ligand, florbetapir. Amyloid positivity on florbetapirPET imaging is correlated with post-mortem $A \beta$ burden, neuritic amyloid plaque density, and neuropathological diagnosis of AD [75]. After intravenous cannulation, $370 \mathrm{MBq}$ florbetapir F18 (Amyvid) is injected. PET data are acquired continuously during and following injection to allow florbetapir uptake dynamics to be assessed. Final amyloid burden is assessed over a 10-min period, $\sim 50 \mathrm{~min}$ after injection, with scope for the previous 10-min period to be used if longer scan periods are not tolerated. PET data, acquired in list-mode, is reconstructed using a 3D ordered-subset expectation-maximisation algorithm with three iterations and 21 subsets, and smoothed with a $4 \mathrm{~mm}$ Gaussian kernel. Attenuation maps are computed by default from the ultra-short echo time (UTE) sequences provided by the vendor as well as from the $\mathrm{T} 1$-weighted and T2-weighted volumetric scans using a multi-atlas CT synthesis method [76], also known as pseudo-CT (pCT). The latter approach significantly improves PET reconstruction accuracy when compared to the UTE-based correction [77], as demonstrated in Fig. 3. 


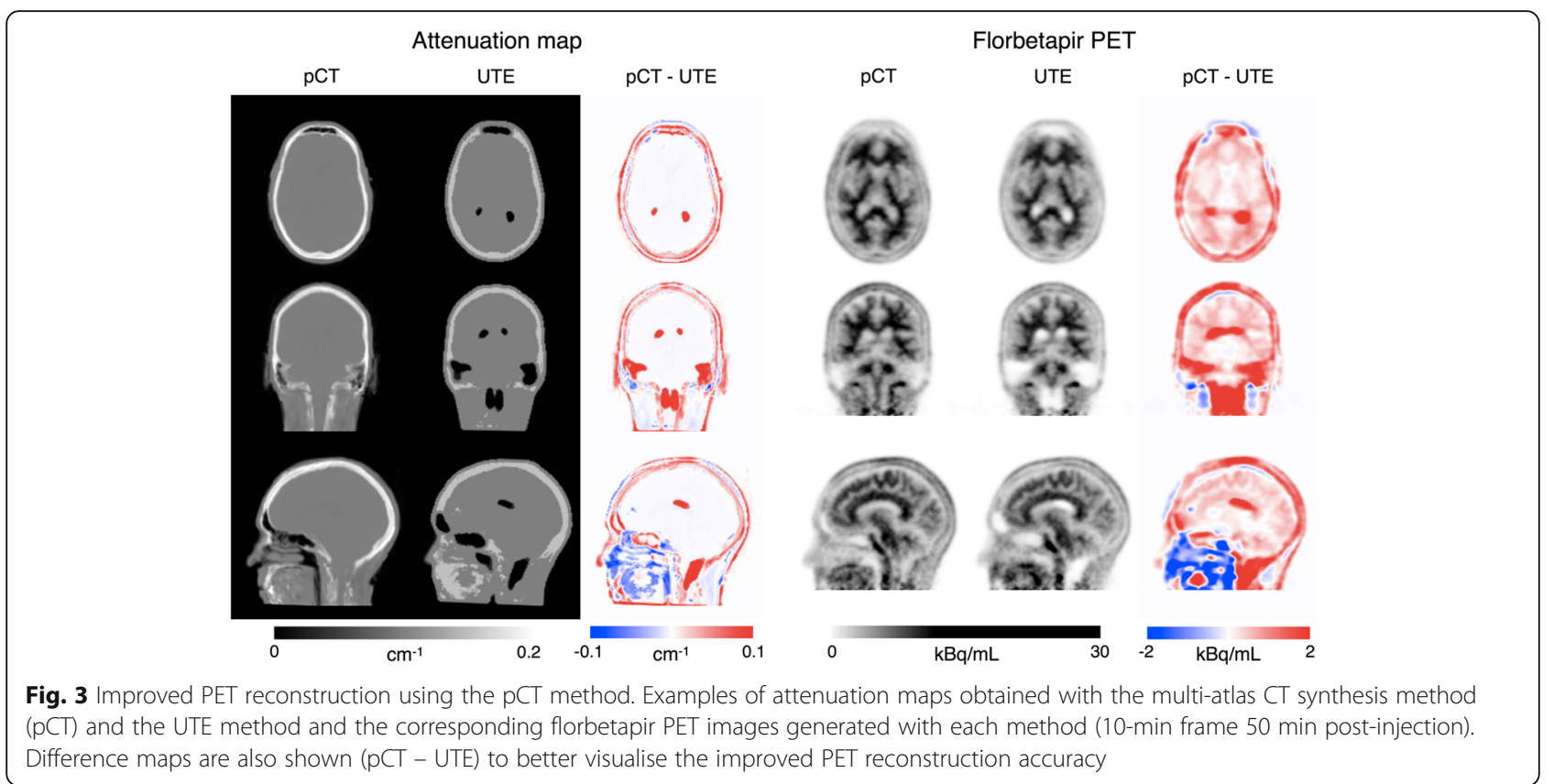

For the MR acquisitions, a body coil RF transmitter is used in conjunction with a 12-channel receiver array head coil. The maximum gradient strength is $45 \mathrm{mT} / \mathrm{m}$ along each direction. The MR sequences are:

(i) high resolution 3D T1-weighted, T2-weighted and FLAIR volumetric scans;

(ii) resting state functional MRI (rs-fMRI);

(iii) multi-shell high angular resolution diffusion-weighted MRI (DW MRI);

(iv) a multi-echo 3D gradient echo sequence for simultaneous T2*-weighted/susceptibility-weighted imaging (SWI), quantitative susceptibility mapping and $\mathrm{b}_{0}$ field mapping; and.

(v) arterial spin labeling (ASL) for quantitative mapping of cerebral blood flow (CBF).

An additional $b_{0}$ field map is also acquired for distortion correction of the rs-fMRI and DW MRI images. Full details of the acquisition parameters are shown in Table 4.

\section{$3 D$ volumetric scans ( $T 1, T 2$ and FLAIR)}

Three volumetric scans are acquired with matched spatial coverage, resolution and complementary contrasts, to aid tissue segmentation, delineation of the intracranial vault, and white-matter lesion visualization. 3D T1-weighted images are obtained using an MPRAGE sequence [78]. This is optimized to provide strong contrast between white matter and grey matter and enable quantification of grey matter macroscopic structures in both cortical and subcortical brain regions. 3D T2weighted images use a long echo train turbo spin echo sequence (SPACE) [79]. FLAIR images are acquired using the same SPACE sequence as T2-weighted images but with the addition of an inversion preparation pulse to null signal from cerebrospinal fluid. T2-weighted and FLAIR images are sensitive to white-matter lesions and hyperintensities, which are typically seen in association with cerebrovascular disease.

Images undergo manual $\mathrm{QC}$ in line with protocols developed for commercial trials, by a trained team who assess motion, coverage and other issues. T1 scans are additionally checked specifically for blurring, image wrap-around and contrast problems, and FLAIR for good CSF suppression.

Pre-processing of structural (T1, T2, FLAIR) images is carried out by applying a correction for gradient non-linearity [80] followed by brain-masked (by registration of MNI template to the scan) N4-bias correction [81]. An automated multi-region parcellation of the T1 images is carried out using geodesic information flow (GIF) [82] - demonstrated in Fig. 4. The parcellation is transferred to microstructure, PET, ASL and fMRI maps for the purpose of region-ofinterest (ROI)-based analysis following registration of those images to the T1 image.

\section{Resting state $f M R I$ ( $r s-f M R I)$}

Resting state, or task-free, fMRI (rs-fMRI) allows for assessment of functional cortical connectivity through analysis of resting state networks (i.e. disparate brain 


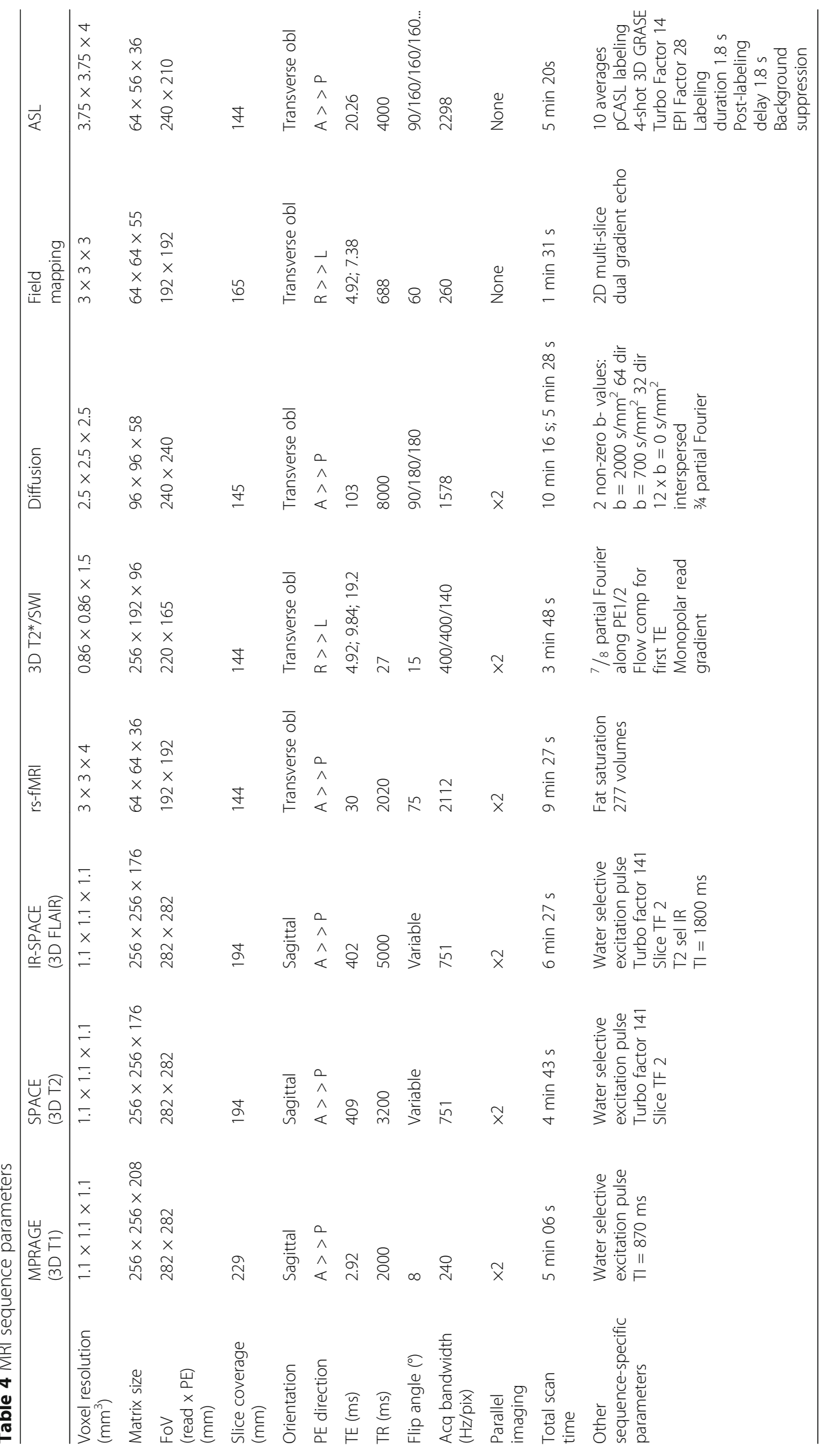




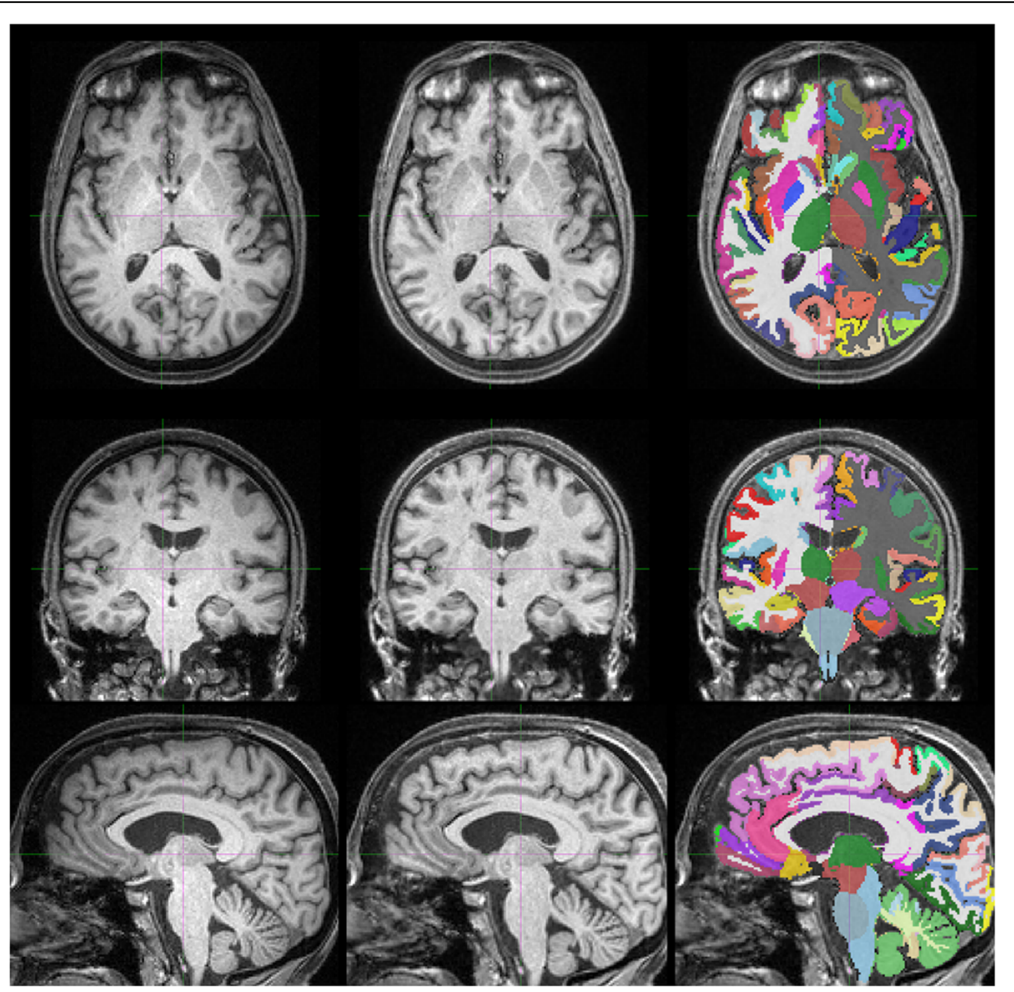

Fig. 4 Volumetric T1 pre-processing and segmentation in Insight 46. Examples of axial (top row), coronal (middle row) and sagittal (bottom row) slices from an original MPRAGE volumetric T1 scan (left column), pre-processed T1 (distortion and bias field corrected) (middle column), and with the GIF parcellation overlaid on top (right column)

regions which exhibit synchronised fluctuations in local cerebral blood flow while the brain is not engaged in any specific task), which are thought to represent cortical networks critical to the functional organisation of the brain [83].

rs-fMRI is acquired using a T2*-weighted gradient echo EPI acquisition scheme. A resolution of $3 \times 3 \times 4 \mathrm{~mm}^{3}$ was chosen to ensure whole brain coverage (defined as $144 \mathrm{~mm}$ coverage in the inferior-superior direction for angled transverse slice orientation [84]) within a reasonable relaxation time (TR) ( $\sim 2$ s). Parallel imaging is also used to accelerate the imaging and reduce image distortions (GRAPPA with acceleration factor 2). At the beginning of the rs-fMRI acquisition, participants are asked to "close their eyes and not to fall asleep" for the duration of the scan.

Individual fMRI time series are visually checked for head coverage, motion, signal dropout and other artefacts. All fMRI volumes are realigned to correct for motion between individual points in the time course. A plot of the registration parameters computed in the motion correction is provided for manual review to ensure that the level of motion is not severe enough to adversely affect the resulting analysis. Signal quality metrics such as signal-to-noise ratio
(SNR), variance of signal change from average signal (DVARs) and spike levels are also plotted over the length of the time course [85]. Timepoints that are outliers are automatically removed from the analysis.

The motion-corrected fMRI time course is then registered to the corresponding anatomical T1 image. A cohort specific group template discretised in MNI space is iteratively computed by mapping all T1 images with 10 (1 rigid, 9 affine) and 10 non-linear registrations into the MNI image space [86]. The fMRI scan is then transformed into the template space by combining the affine registration from fMRI to T1 image with the transformation that maps the individual T1 image into the group template in MNI space. A Generalised Linear Model (optimised with restricted maximum likelihood estimation (REML) [87]) is used to account for signal drifts and physiological noise using cosine basis functions (highpass filtering of frequencies $>0.01 \mathrm{~Hz}$ ), the demeaned motion-realignment estimates and their derivatives, and RETROICOR regressors, where appropriate [88].

Two methods of analyzing the pre-processed data will be used: a seed-based method and independent component analysis (ICA) [89]. In brief, the remaining residuals 
are smoothed (Gaussian smoothing kernel with $5 \mathrm{~mm}$ FWHM) and mapped into the subsampled group space to create spatial correspondence among individual brains. A seed region is chosen to extract an average time course that is correlated with the time course of every individual voxel. The resulting correlation map per participant is Fisher z-transformed to enable t-test hypothesis testing among participants. For the ICA, time courses of the motionrealigned fMRI scan within a mask of the brain are extracted, centered and variance-normalized, resulting in one voxel-time matrix per participant. All participant matrices are then concatenated in time. The obtained group matrix is reduced to its principal components and whitened. The independent component analysis [90] is applied to the whitened group matrix to obtain spatial components. The representation of all groupindependent components in each participant is required for group comparison. Dual regression will be applied to obtain group-independent component representations in each participant.

\section{Diffusion-weighted MRI}

Diffusion-weighted MRI is a technique that enables characterisation of the microstructural integrity of white and grey matter. The majority of studies investigating neurodegeneration utilise the diffusion tensor model [91], which aggregates the differential diffusion profiles of water molecules in extra- and intracellular spaces to produce an array of metrics including fractional anisotropy (FA), axial diffusivity (AxD), radial diffusivity (RD) and mean diffusivity (MD). These metrics have been used to demonstrate change in white-matter tract integrity and grey-matter microstructure in AD [92]. However, this model does not account for multiple fiber orientations or tissue compartments within a voxel, making interpretation of changes in these metrics ambiguous. Neurite orientation dispersion and density imaging (NODDI) [93] is a recently-developed multi-shell diffusion technique which allows for the estimation of tissue microstructure at the sub-voxel level by assuming that water protons in neuronal tissue can be considered to be in three different pools: i) free water, modelling CSF space; ii) restricted water, modelling dendrites and axons; and iii) hindered water, modelling diffusion within glial cells, neuronal cell bodies and the extracellular environment. This more complex modelling enables estimation of neurite density (neurite density index (NDI)) and neurite orientation dispersion (orientation dispersion index (ODI)) in both white and grey matter.

Diffusion MRI is acquired using a twice-refocused spin echo EPI sequence [94, 95] with two non-zero bvalues (700 and $2000 \mathrm{~s} / \mathrm{mm}^{2}$ ) and multiple directions
(32 and 64 directions for the $\mathrm{b}=700 / 2000 \mathrm{~s} / \mathrm{mm}^{2}$ scans respectively). The b-vector directions were calculated to be uniformly distributed over a hemisphere, and images with $\mathrm{b}=0 \mathrm{~s} / \mathrm{mm}^{2}$ are interspersed throughout the acquisition (12 obtained overall). Images are acquired with an isotropic $2.5 \times 2.5 \times 2.5 \mathrm{~mm}^{3}$ resolution, with 58 slices to ensure whole brain coverage.

Visual review is performed for identification of poor quality images by checking for: (i) full brain coverage; (ii) inter-acquisition motion (using motion plots over the acquisition); (iii) sufficient correction of geometric distortion; and (iv) slice-wise signal dropout (using correlation plots between adjacent slices). Images failing this quality-control process are removed before running the diffusion analysis. For each participant, if the number of acquisitions that have failed is high enough that it might affect the consistency of the analysis between subjects, then these data are marked as a 'failed acquisition'.

Pre-processing of diffusion-weighted images involves first correcting for inter-volume motion registration and eddy currents using FSL's Eddy tool [96]. This is followed by correction for EPI susceptibility distortion using field maps to the structural T1 [97] and phaseencode direction constrained non -linear registration to the T1 volume, with modulation based on the Jacobian determinants. The separate diffusionweighted shells (together with their associated $b=0$ volumes) are fitted with a diffusion tensor model using NiftyFit [98]. The NODDI model is then fitted to the combined shells [93] (see Fig. 5 for an example of the diffusion-weighted images and corresponding parameter maps).

\section{D T2*/SWI}

T2\%/SWI are iron-sensitive sequences that allow for detection of cerebral microbleeds, associated with cerebral small vessel disease or cerebral amyloid angiopathy, generally distinguished by their distribution within the brain parenchyma. Microbleeds are more common in AD than controls [99], and may independently impact on cognitive function with potential mechanisms including tissue necrosis in strategic white-matter tracts [100], or via the initiation of an inflammatory cascade [100].

A 3D multi-echo gradient echo sequence is acquired to generate $\mathrm{T} 2 *$-weighted images, $\mathrm{T} 2 *$ maps, SWI, and quantitative susceptibility maps (qSM). This sequence can also provide $b_{0}$ field mapping at higher resolution than the GRE-based field maps that are currently often used for processing geometric distortion correction of the DWI and fMRI. The sequence acquires magnitude and phase images at three echo 


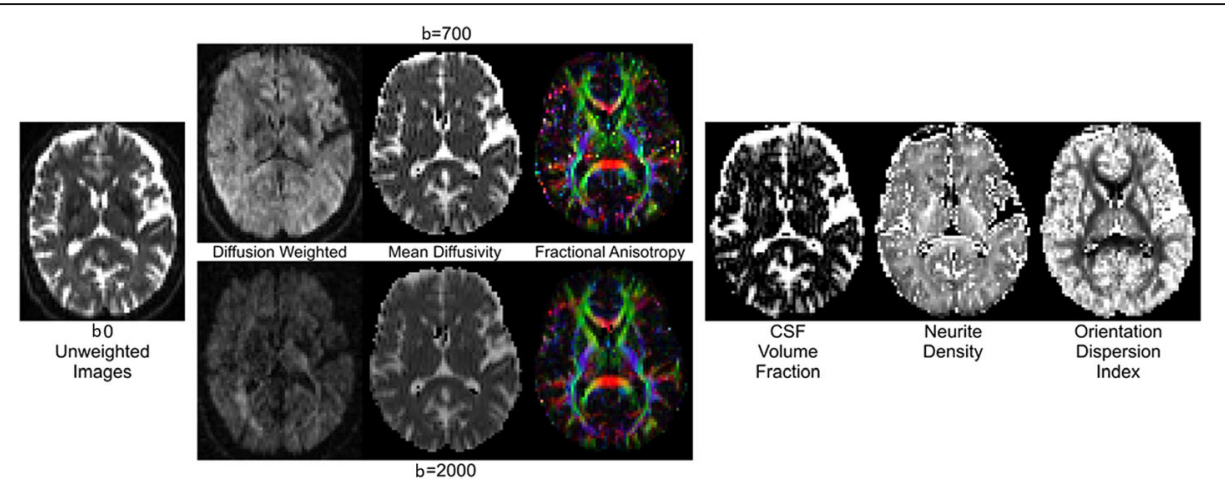

Fig. 5 Representative diffusion images in Insight 46. Examples of diffusion images at the two b values, b = 700 and $2000 \mathrm{~s} / \mathrm{mm}^{2}$, with their corresponding derived MD and FA maps (left) and NODDI metrics (right)

times: $\mathrm{TE}=4.92,9.84$, and $19.7 \mathrm{~ms}$ (chosen to keep fat and water signals in phase). Magnitude images from the longest TE $(19.7 \mathrm{~ms})$ provide the T2\%weighted volume, which are combined with the phase images from the same echo time to generate SWI [101]. T2* maps are generated by fitting the three magnitude images to a monoexponential decay $\mathrm{S}=\mathrm{S} 0$.exp. $(-\mathrm{TE} / \mathrm{T} 2 *)$, and $\mathrm{qSM}$ are obtained from the three phase images using the superfast dipole inversion (SDI) method [102]. The images are visually checked for coverage, motion, and artefacts.

\section{Arterial spin labelling (ASL)}

ASL is a non-invasive method for determining regional cerebral blood flow (CBF). In brief, blood flowing through the neck vessels is labelled via spin inversion. After a delay period to allow this labelled blood to perfuse into the brain, images are acquired, and compared with equivalent images acquired without prior spin labelling (unlabelled images). The signal difference between labelled and unlabelled images is proportional to blood flow, thus enabling calculation of a quantitative cerebral blood flow map.

ASL data are acquired using a 4-shot segmented 3D GRASE pCASL sequence [103] (for more details refer to Table 4). The ASL timing parameters were chosen based on the recommendations of the recent ISMRM Perfusion Study Group consensus paper [104]: labeling duration $=1800 \mathrm{~ms}$; post labelling delay $=1800 \mathrm{~ms}$. Ten averages are acquired and saved separately, in order to allow image realignment prior to averaging. Background suppression is used to reduce static tissue signal, and an accompanying set of three saturation recovery data sets $(\mathrm{TR}=1 \mathrm{~s}, 2 \mathrm{~s}$, $/ 2 / 4$ s) are acquired using the same 3D GRASE acquisition scheme for estimation of tissue T1 and M0 (total scan time $\sim 50 \mathrm{~s}$ ).
During pre-processing, CBF maps are calculated using the recommended model for ASL images acquired with a single post-labelling delay [104], implemented in NiftyFit [98] and assuming blood $\mathrm{T} 1$ at $3 \mathrm{~T}$ of $1650 \mathrm{~ms}$ and an overall labelling efficiency of 0.833 (blood inversion efficiency of 0.85 and two background suppression pulses with inversion efficiency 0.99 each). The saturation recovery data are fitted to a monoexponential recovery curve to estimate the underlying tissue magnetisation (M0) and receiver coil sensitivity variation, enabling generation of quantitative CBF maps [98]. Error maps are also provided to allow inference of how precisely $\mathrm{CBF}$ in different regions has been estimated.

\section{Primary imaging pipelines and analyses}

All imaging data, derived results, visual quality checks, and radiological reads are stored on a customised web-based server running XNAT 1.6.5 (www.xnat.org). As protected health information (PHI) is stored in the proprietary listmode format, but difficult to remove, the decision was made not to enter any PHI on the scanner console of these participants. After data are acquired on the PET/MR scanner they are transferred to a study-specific waypoint. This includes both the DICOM-compliant imaging format used for MRI and PET images that are reconstructed on the scanner and raw listmode data that consists of a customised DICOM file which contains the Interfile header and a corresponding binary data file generated from the full $60 \mathrm{~min}$ of PET acquisition. Twice daily, all recent data are securely synchronised between the study-specific waypoint and the XNAT server, where separate import processes are done for both the DICOM and listmode data. DICOM data are checked for completeness before formally importing the data into the 
XNAT database. Once the DICOM data have been imported, listmode data are then added to the PETMR imaging session in the database and automatic checks are performed to ensure that they contain the full 60-min acquisition, including some small amount of time before the tracer was injected. Next, an in-house workflow management system automatically starts the modality-specific pre-processing steps mentioned in the previous section. Key derived images generated during these pre-processing steps are stored on the XNAT server by attaching them to the original imaging session. Once pre-processing is complete, visual review for each modality is performed and a customised modality-specific webform of the quality checking is stored on the XNAT server.

A consultant neuroradiologist reviews all T1, T2 and FLAIR sequences as outlined in the "Duty of Care" section by downloading the key imaging data from the server and completing a customised radiological read web-form within XNAT. Customised reports are provided to the neuroradiologist which identify the PET-MR imaging sessions where a read needs to be completed.

Primary analyses of T1 images include automated segmentation of whole-brain [105] and hippocampal regions [106], followed by manual checking and editing, semi-automated ventricle segmentation, automated total incracranial volume (TIV) measurement [107] and semiautomated cortical thickness calculation [108]. Figure 4 provides an example of the GIF segmentation and Fig. 6 shows summary volumetric metrics from the first $100 \mathrm{~T} 1$ scans. White-matter burden and micro haemorrhages are assessed using visual rating scales $[109,110]$, and automated quantification $[111,112]$.

Volume loss between serial scans will be determined using the robust boundary shift integral (BSI) [113] following affine registration of repeat and baseline scans. Regional BSI calculation is performed using a fully affine whole-brain registration followed by rigid registration of masked local structures. Tensor-based morphometry is used as a non-region-based measure of volume loss.

The amyloid PET images are registered to the T1-scan and visually rated as positive/negative by experienced nuclear medicine specialists [114]. Standardised uptake value ratios (SUVR) are calculated globally and locally from a T1-parcellation [115]. Regional grey matter amyloid load is calculated with appropriate partial volume correction [116].

\section{Blood and urine specimen protocol for clinical samples and biomarker identification}

Blood samples are collected for haemoglobin, platelet count, urea and creatinine, random glucose, vitamin $\mathrm{B} 12$, and TSH, as potential modulators both of cognition and progression of Alzheimer's pathology. Samples will also be stored for biomarker exploration (both serum and plasma) and genetic analysis. Blood is collected using a Vacutainer system in a single venepuncture procedure. Samples are inverted

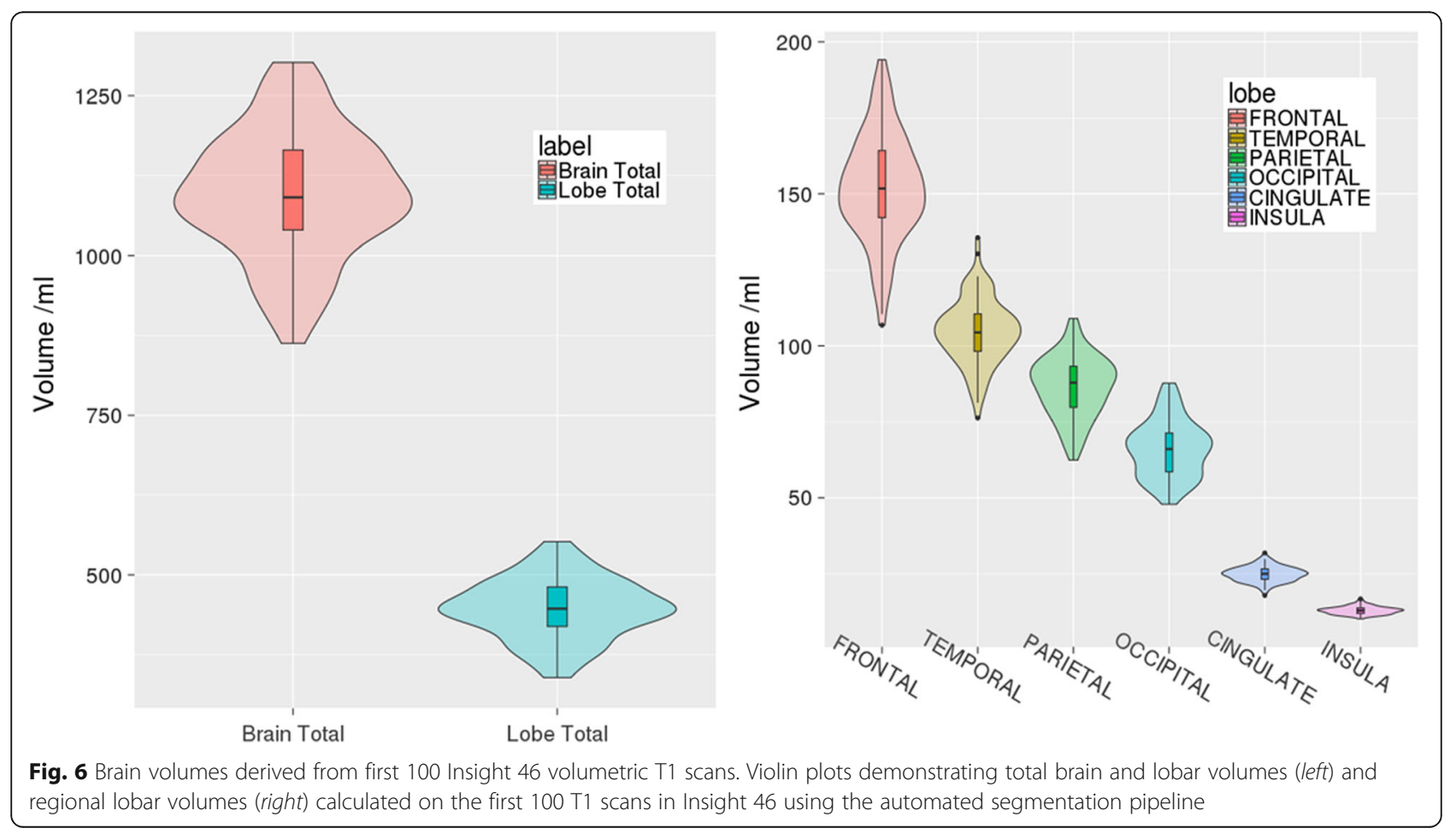


eight times to ensure adequate mixing of blood with tube additives. All samples are processed within two hours of collection, as per recent working group guidelines on biomarker pre-processing [117]. One $4.0 \mathrm{ml}$ EDTA sample is collected for haemoglobin and platelet count and the remaining sample is stored at $-20{ }^{\circ} \mathrm{C}$ prior to genetic extraction. One $2.5 \mathrm{ml} \mathrm{SST} \mathrm{sample} \mathrm{is} \mathrm{collected} \mathrm{for} \mathrm{urea,} \mathrm{creatinine,}$ TSH, vitamin B12 and random glucose. Two $10 \mathrm{ml}$ EDTA samples and two clotted $8.5 \mathrm{ml}$ SST samples are spun at $2000 \mathrm{~g}$ for $10 \mathrm{~min}$ to generate up to $8.0 \mathrm{ml}$ plasma stored as $16 \times 0.5 \mathrm{ml}$ aliquots and up to $7.0 \mathrm{ml}$ serum stored as $14 \times 0.5 \mathrm{ml}$ aliquots, all at $-80{ }^{\circ} \mathrm{C}$, for later analysis. Aliqot tubes are made from polypropylene. Urine is collected in a $100 \mathrm{ml}$ polypropylene pot and transferred on ice for storage. A total of $24 \mathrm{ml}$ urine is stored across 5 aliquots at $-80{ }^{\circ} \mathrm{C}$. Planned analyses include measurement of serum neurofilament light [118], plasma tau [119] and plasma $A \beta 40$ and $A \beta 42$ [120] using ultrasensitive Single molecule array (Simoa) assays [121].

\section{Genetics}

DNA from each participant is extracted from whole blood using standard methods (phenol-chloroform). Existing genotyping data are available from the Metabochip [122] and the DrugDev Consortium Array (Illumina, inc.) and samples are further assayed on NeuroX2 (Infinium NeuroConsortium Array, Illumina, inc.) according to the recommended protocol. This genotyping platform is the second iteration of a neurological disease-specific array. It covers approximately 500,000 genetic markers, many of which will have a role in neurodegenerative disease, and improves on NeuroX which was designed and released in 2014 [123]. NeuroX2 includes an up-to-date content, with the latest signals from the most recent genome-wide association study (GWAS) in neurodegenerative diseases, such as PD and AD. As an example, all known genome-wide associated and suggestive loci for $\mathrm{AD}$ are present in the array, which enables the creation and ascertainment of polygenic risk scores for that disease [15], which can then be improved by using biomarker and imaging data from the same participants.

\section{Analysis plan}

Overarching themes of the study include better characterisation of the prevalence and incidence of cerebral amyloidosis in a British birth cohort; examining how biological, genetic, psychological and social factors across life influence cerebrovascular burden, amyloid burden, and neurodegeneration (as measured by cortical network breakdown and cerebral atrophy), and their interplay on cognition; and in doing so establishing metrics that are sensitive for detecting early neurodegeneration.

Specific research questions we will address include: the extent and variability of amyloid deposition and proportion of this representative cohort who will be amyloid positive (estimated at 15-25\%); the relationships between amyloid load, standard and more advanced MR metrics and vascular burden; the influence of childhood cognitive and motor ability, educational attainment, lifetime mental health, physical activity, physical function and cerebrovascular risk profiles and genetic factors on the development of brain pathology, cognitive health, gait and motor skills; the cognitive tests - both established and novel - that are most associated with cross-sectional biomarkers of brain pathology and have most power to detect change over time and thus to be outcome measures for clinical trials; the extent to which genetic risk scores and blood-based biomarkers can detect asymptomatic amyloidosis; and how best to recruit to presymptomatic $\mathrm{AD}$ trials and which imaging and other biomarkers will maximise power to detect treatment effects in the preclinical and very early stages of cognitive decline.

\section{Discussion}

Insight 46 intends to integrate the NSHD data on the socioeconomic environment and on physical, psychological and cognitive function from 0 to 69 years, coupled with data on genetics, structural and molecular imaging, and intensive cognitive and neurological phenotyping, to inform what influences the entire spectrum of changes that occur as the brain ages: from healthy through to pathological ageing, with a specific focus on $\mathrm{AD}$. Combining the cohort's uniquely rich life course data with the metrics collected in Insight 46, at an age when overt dementia is rare, provides an unprecedented opportunity to explore pre-symptomatic neurodegeneration and to evaluate very subtle cognitive decline. Prospective follow up in the sub-study allows for the consequences of these changes to be quantified. These analyses, initially performed in house, with subsequent data dissemination in line with the MRC Policy on data sharing, have the potential to provide fundamental insights into the factors that influence healthy and pathological brain ageing, provide an evidence base to inform how best to identify individuals at high risk for $\mathrm{AD}$ and other forms of dementia, and contribute to practices for monitoring change over time for clinical trials. 


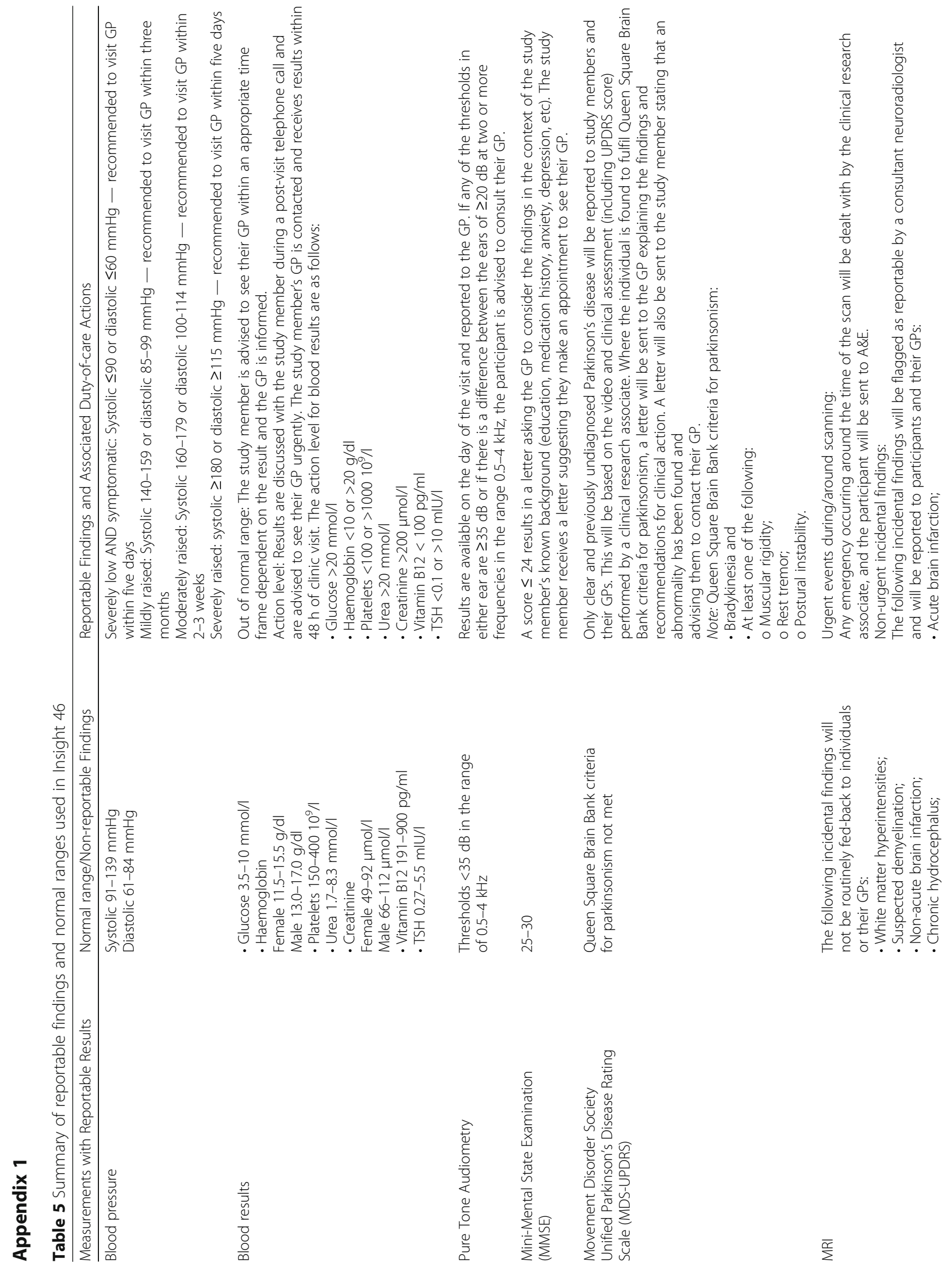




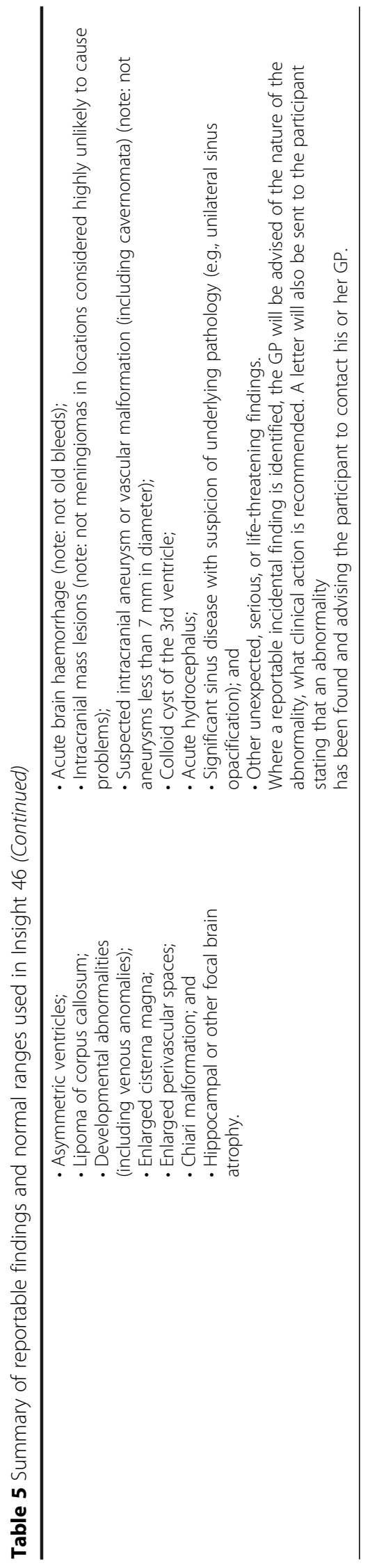




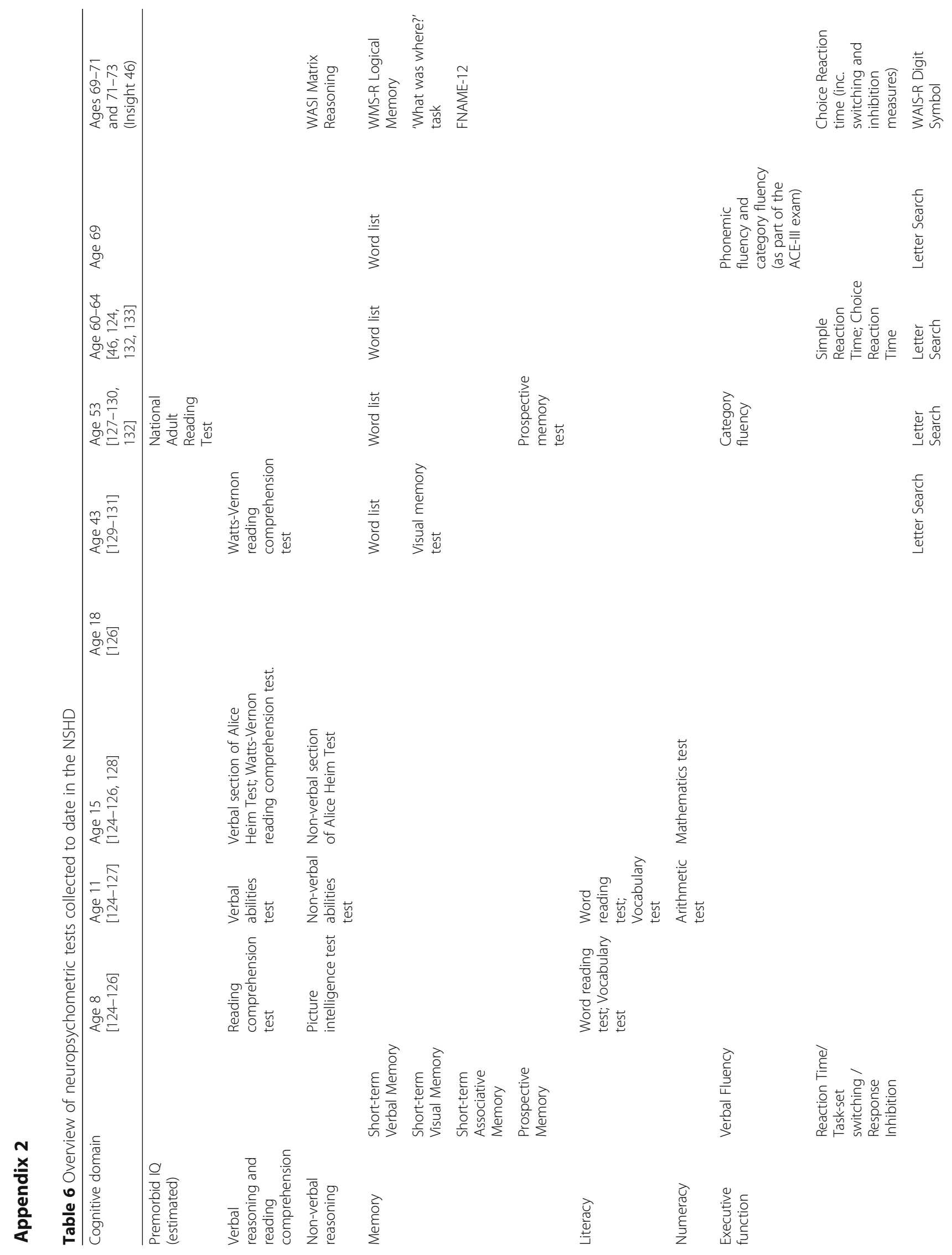




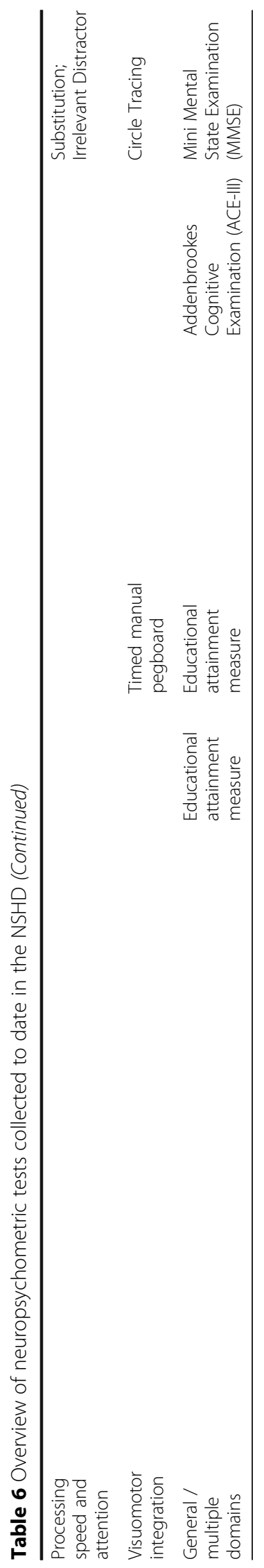




\section{Abbreviations}

3 T: 3 Tesla (magnetic field strength); ACE-III: Addenbrooke's Cognitive Examination 3rd revision; AD: Alzheimer's disease; ASL: arterial spin labelling; AxD: axial diffusivity; $A B$ : beta-amyloid; BRAIN: BRadykinesia Akinesia INcoordination test; CBF: cerebral blood flow; CDR: Clinical Dementia Rating scale; DICOM: Digital Imaging and Communications in Medicine, a standard for handling, storing, printing, and transmitting information in medical imaging; DVAR: variance of signal change from average signal in fMRI; DW MRI: diffusion-weighted MRI; EDTA: ethylenediaminetetraacetic acid; EPI: echo planar imaging; FA: fractional anisotropy; FLAIR: fluid attenuated inversion recovery MRl; fMRI: functional MRI; FNAME-12A: 12-item Face-Name Associative Memory Exam; FNAME-16: 16-item Face-Name Associative Memory Exam; FWHM: full width at half maximum (spatial resolution); GIF: geodesic information flow, an in-house method of MRI segmentation; GRAPPA: GeneRalized Autocalibrating Partial Parallel Acquisition, an MRI multi-coil parallel imaging technique; GRASE: gradient- and spin-echo MRI; GWAS: genome wide association study; ICA: independent component analysis; MCl: mild cognitive impairment; MD: mean diffusivity; MMSE: mini-mental state examination; MPRAGE: magnetisation prepared rapid gradient echo MRI; MRC: Medical Research Council; MRI: magnetic resonance imaging; NDI: neurite density index; NODDI: neurite orientation dispersion and density index; NRES: National Research Ethics Service; NSHD: National Survey of Health and Development; ODI: orientation dispersion index; pCASL: pseudo-Continuous Arterial Spin Labelling; PD: Parkinson's disease; PEEK: Portable Eye Examination Kit; PET: positron emission tomography; PHI: protected health information; QC: quality control; RD: radial diffusivity; REC: research ethics committee; REM: rapid eye movement; SCD-Q: Subjective Cognitive Decline Questionnaire; SNR: signal-to-noise ratio; SPACE: Sampling Perfection with Application optimized Contrasts using different flip angle Evolution MRI; SST: serum-separator tube; SUVR: standardised uptake value ratio; T1: MRI spin-lattice or longitudinal relaxation time; T2*: MRI measure of loss of phase coherence among spins oriented at an angle to the static magnetic field due to a combination of magnetic field inhomogeneities and the spin-spin relaxation; T2: MRI spin-spin or transverse relaxation time; TE: MRI echo time; TR: MRI repetition time; TSH: thyroid-stimulating hormone; UCL: University College London; UPDRS: Unified Parkinson's Disease Rating Scale; UPSIT: University of Pennsylvania Smell Identification Test; UTE: MRI ultrashort TE; WAIS-R: Wechsler Adult Intelligence Scale-Revised; WASI: Wechsler Abbreviated Scale of Intelligence; WMS-R: Wechsler Memory Scale-Revised; XNAT: an open-source imaging informatics software platform

\section{Acknowledgements}

We are very grateful to those study members who helped in the design of the study through focus groups, and to the participants both for their contributions to Insight 46 and for their commitments to research over the last seven decades. We are grateful to the radiographers and nuclear medicine physicians (Professor Ashley Groves, Dr. Jamshed Bomanji, Dr. Irfan Kayani) at the UCL Institute of Nuclear Medicine, and to the staff at the Leonard Wolfson Experimental Neurology Centre at UCL. We acknowledge the help of the following collaborators for their assistance with specific tests included in the protocol, including Dr. Patrick Esser, Dr. Emily Henderson and Professor Helen Dawes (gait task); Professor Masud Husain and Dr. Yoni Pertzov (What was where?' visual short-term memory binding task); Professor Julie Stout (visuomotor integration task); Professor Nilli Lavie (irrelevant distractor paradigm); Dr. Dorene Rentz, Dr. Kathryn Papp and Dr. Reisa Sperling (FNAME-12); Dr. Alastair Noyce (BRAIN test); Dr. Doris Bamiou, Professor Jason Warren, Professor Stuart Rosen and Dr. Tim Schoof (auditory tasks); Jamie Toombs (wet biomarker preprocessing); Professor Rebecca Hardy for advice on sample selection and statistical methods; and to Professor Yoav Ben Shlomo and Professor Aroon Hingorani for advice on gait/ parkinsonism and genetics respectively. We would like to acknowledge Dan Marcus and Rick Herrick for assistance with XNAT, Dr. Philip Curran for assistance with data sharing with the MRC Unit for Lifelong Health and Ageing, the DRC trials team for assistance with imaging QC, and Mark White for his work on data connectivity. This study is principally funded by grants from Alzheimer's Research UK (ARUK-PG2014-1946, ARUK-PG2017-1946), the Medical Research Council Dementias Platform UK (CSUB19166), and the Wolfson Foundation (PR/ylr/18575). The genetic analyses are funded by the Brain Research Trust (UCC14191). Florbetapir amyoid tracer is kindly provided by AVID Radiopharmaceuticals (a wholly owned subsidiary of Eli Lilly) who had no part in the design of the study: we are particularly indebted to the support of the late Dr. Chris Clarke of AVID Radiopharmaceuticals who championed this study from its outset. The NSHD, DK,
MR, NS and AW are funded by the Medical Research Council (MC_UU_12019/1, MC_UU_12019/3). Some researchers are supported by the NIHR Queen Square Dementia BRU (JMS, NCF, SMDH), UCL Hospitals Biomedical Research Centre (JMS,EDV), Leonard Wolfson Experimental Neurology Centre (JMS, NCF, MM, DT). TP is supported by a Wellcome Trust Clinical Research Fellowship (200109/ Z/15/Z). DMC is supported by an Alzheimer's Society Project Grant (AS-PG-205). MM is supported by an Alzheimer's Society Project Grant (AS-PG-15-025). AM is supported by an MRC grant (MR/S01 107X/1). CS is supported by an MRC platform grant (EP/M020533/1). SC is supported by an Alzheimer's Research UK Senior Research Fellowship (ARUK-SRF2013-8). JH receives grant support from an Anonymous Foundation. SO receives funding from the EPSRC (EP/H046410/ 1, EP/J020990/1, EP/K005278), the MRC (MR/J01107X/1), the EU-FP7 project VPH-DARE@IT (FP7-ICT-2011-9-601,055), and NIHR BRC UCLH/UCL High Impact Initiative (BW.mn.BRC10269). NCF is supported by an NIHR Senior Investigator award, and additional funding from the NIA and EPSRC. JMS acknowledges the EPSRC (EP/J020990/1) and European Union's Horizon 2020 research and innovation programme (Grant 666,992).

\section{Funding}

Insight 46 is funded by grants from Alzheimer's Research UK (ARUK-PG2014-1946, ARUK-PG2017-1946 PIs Schott, Fox, Richards), the Medical Research Council Dementias Platform UK (CSUB19166 PIs Schott, Fox, Richards), the Wolfson Foundation (PR/ylr/18575 PIs Fox, Schott), the Medical Research Council (MC_UU_12019/ 1 PI Kuh and MC_UU_12019/3 PI Richards), the Wellcome Trust (Clinical Research Fellowship 200,109/Z/15/Z Parker) and Brain Research Trust (UCC14191, PI Schott). AVID Radiopharmaceuticals (a wholly owned subsidiary of Eli Lilly) provide the PET amyloid tracer (Florbetapir) but had no part in the design of the study.

\section{Availability of data and material}

Data generated in Insight 46 will be made available one year after each wave of data collection is completed, allowing sufficient time for quality control, and confidentiality of imaging data to be assured. Data will be made available to bona fide researchers through LHA's standard security processes, and according to established NSHD data sharing guidelines. Requests for data will be considered by a data sharing committee on the basis of quality, scientific priorities and overlapping interests.

\section{Authors' contributions}

CAL, TP, DMC, KM, ED, HM-S wrote parts of the first draft and contributed to the overall design of the sub-study. AB, DBr, DBe, DMC, NB, MJC, AC, EDV, JD, MH, ME, $J \mathrm{~K}, \mathrm{IM}, \mathrm{PM}, \mathrm{AM}, \mathrm{MM}, \mathrm{CS}, \mathrm{DT}, \mathrm{HZh}$, and SO contributed to the design of the imaging protocols and analysis pipelines, and critically read the manuscript. ED, KM, JC, SMDH, SC designed the neuropsychology protocols, and critically read the manuscript. HM-S, MB, SB, NE, AW contributed to sub-study design, planned the data management, and critically read the manuscript. JB, AW, JH planned the genetic analysis plans, and critically read the manuscript. CH, NS and SS designed the imaging duty of care plan and critically read the manuscript. NS and AS planned the movement disorders and gait protocols, and critically read the manuscript. HZe planned the wet biomarker analysis plans, and critically read the manuscript. DK directs the NSHD, planned the sub-study and critically revised drafts of this paper. MR and NCF planned the sub-study, obtained funding, managed the sub-study, and critically revised drafts of this paper. JMS planned the sub-study, obtained funding, managed the sub-study, drafted aspects of the paper, and is the guarantor. All authors read and approved the final manuscript.

\section{Competing interests}

HZe is a co-founder of Brain Biomarker Solutions, a GU venture-based platform company at the University of Gothenburg and has served on advisory boards of Eli Lilly, Roche Diagnostics and Pharmasum Therapeutics. JD is a consultant for GE Healthcare and Bioclinica. NCF's research group has received payment for consultancy or for conducting studies from AVID, Bristol-Myers Squibb, Elan Pharmaceuticals, Eisai, Lilly Research Laboratories, GE Healthcare, IXICO, Janssen Alzheimer Immunotherapy, Johnson \& Johnson, Janssen-Cilig, Lundbeck, Neurochem Inc., Pfizer Inc., Sanofi-Aventis and Wyeth Pharmaceuticals. NCF receives no personal compensation for the activities mentioned above. JS has received research funding from Avid Radiopharmaceuticals (a wholly owned subsidiary of Eli Lilly), has consulted for Roche Pharmaceuticals and Eli Lilly, and serves on a Data Safety Monitoring Committee for Axon Neuroscience SE.

\section{Consent for publication}

Not applicable. 


\section{Ethics approval and consent to participate}

Ethical approval for the neuroscience sub-study was granted by the National Research Ethics Service (NRES) Committee London (REC reference 14/LO/1173, PI Schott). All participants provide written informed consent to participate and for their data to be stored in accordance with the Data Protection Act.

\section{Publisher's Note}

Springer Nature remains neutral with regard to jurisdictional claims in published maps and institutional affiliations.

\begin{abstract}
Author details
'Dementia Research Centre, Institute of Neurology, University College London, London, UK. ${ }^{2}$ Translational Imaging Group, Centre for Medical Image Computing, University College London, London, UK. ${ }^{3}$ Leonard Wolfson Experimental Neurology Centre, Institute of Neurology, University College London, London, UK. ${ }^{4}$ Institute of Nuclear Medicine, University College London Hospitals, London, UK. ${ }^{5}$ Department of Molecular Neuroscience, Institute of Neurology, University College London, London, UK. ${ }^{6}$ Department of Medical Sciences and Institute of Biomedicine - iBiMED, University of Aveiro, Aveiro, Portugal. ${ }^{7}$ MRC Unit for Lifelong Health and Ageing at UCL, London, UK. ${ }^{8}$ Lysholm Department of Neuroradiology, The National Hospital for Neurology and Neurosurgery, Queen Square, London, UK.

${ }^{9}$ Neuroradiological Academic Unit, Department of Brain Repair and Rehabilitation, Institute of Neurology, University College London, London, UK. ${ }^{10}$ Department of Clinical Neuroscience, Institute of Neurology, University College London, London, UK. ${ }^{11}$ National Hospital for Neurology and Neurosurgery, London, UK. ${ }^{12}$ Department of Computer Science and Centre for Medical Image Computing, University College London, London, UK.

${ }^{13}$ Reta Lila Weston Research Laboratories, Department of Molecular Neuroscience, Institute of Neurology, University College London, London, UK ${ }^{14}$ Department of Psychiatry and Neurochemistry, Institute of Neuroscience and Physiology, the Sahlgrenska Academy at the University of Gothenburg, Mölndal, Sweden. ${ }^{15}$ Clinical Neurochemistry Laboratory, Sahlgrenska University Hospital, Mölndal, Sweden.
\end{abstract}

Received: 11 January 2017 Accepted: 21 March 2017

Published online: 18 April 2017

\section{References}

1. Office of National Statistics. Deaths Registered in England and Wales; 2016. p. 1-15.

2. Prince M, Albanese $E$, Guerchet M, Prina M, Richard Pender C, Ferri C, et al. World Alzheimer. Report 2014 Dementia and Risk Reduction an Analysis of Protective and Modifiable Factors. 2014.

3. Alzheimer's Association. Changing the Trajectory of Alzheimer's Disease: A National Imperative. Alzheimer's Assoc. 2010:1-17.

4. Braak H, Braak E. Frequency of stages of Alzheimer-related lesions in different age categories. Neurobiol Aging. 1997;18:351-7.

5. Bateman RJ, Xiong C, Benzinger TLS, Fagan AM, Goate A, Fox NC, et al Clinical and biomarker changes in dominantly inherited Alzheimer's disease. N Engl J Med. 2012;367:795-804.

6. Villemagne VL. Burnham S, Bourgeat P, Brown B, Ellis $K$ a, Salvado O, et al. Amyloid $\beta$ deposition, neurodegeneration, and cognitive decline in sporadic Alzheimer's disease: a prospective cohort study. Lancet Neurol. 2013;12:357-67.

7. Sperling RA, Aisen PS, Beckett LA, Bennett DA, Craft S, Fagan AM, et al. Toward defining the preclinical stages of Alzheimer's disease: recommendations from the National Institute on Aging-Alzheimer's association workgroups on diagnostic guidelines for Alzheimer's disease. Alzheimers Dement. 2011;7:280-92.

8. Jack CR, Wiste HJ, Weigand SD, Rocca WA, Knopman DS, Mielke MM, et al. Age-specifi c population frequencies of cerebral $\beta$-amyloidosis and neurodegeneration among people with normal cognitive function aged 50-89 years: a cross- sectional study. Lancet Neurol 2014;13:997-1005.

9. Dubois B, Feldman HH, Jacova C, Hampel H, Molinuevo JL, Blennow K, et al. Advancing research diagnostic criteria for Alzheimer's disease: the IWG-2 criteria. Lancet Neurol. 2014;13:614-29.
10. Reiman EM, Langbaum JBS, Fleisher AS, Caselli RJ, Chen K, Ayutyanont N, et al. Alzheimer's prevention Initiative: a plan to accelerate the evaluation of presymptomatic treatments. J Alzheimers Dis. 2011;26(Suppl 3):321-9.

11. Mills SM, Mallmann J, Santacruz AM, Fuqua A, Carril M, Aisen PS, et al. Preclinical trials in autosomal dominant AD: implementation of the DIAN-TU trial. Rev Neurol. 2013;169:737-43.

12. Sperling RA, Rentz DM, Johnson KA, Karlawish J, Donohue M, Salmon DP, et al. The A4 study: stopping AD before symptoms begin? Sci Transl Med. 2014;6: $228 \mathrm{fs} 13$.

13. Mormino EC. The relevance of Beta-Amyloid on markers of Alzheimer's disease in clinically normal individuals and factors that influence these associations. Neuropsychol Rev. 2014:300-12.

14. Brodaty $\mathrm{H}$, Mothakunnel $\mathrm{A}$, de Vel-Palumbo $\mathrm{M}$, Ames $\mathrm{D}$, Ellis KA Reppermund S, et al. Influence of population versus convenience sampling on sample characteristics in studies of cognitive aging. Ann Epidemiol. 2014;24:63-71.

15. Escott-Price V, Sims R, Bannister C, Harold D, Vronskaya M, Majounie E, et al. Common polygenic variation enhances risk prediction for Alzheimer's disease. Brain. 2015;138:3673-84.

16. Xu W, Tan L, Wang H-F, Jiang T, Tan M-S, Tan L, et al. Meta-analysis of modifiable risk factors for Alzheimer's disease. J Neurol Neurosurg Psychiatry. 2015;86:1299-306.

17. Kuh D, Pierce M, Adams J, Deanfield J, Ekelund U, Friberg P, et al. Cohort profile: updating the cohort profile for the MRC National Survey of health and development: a new clinic-based data collection for ageing research. Int J Epidemiol. 2011:40

18. Wadsworth M, Kuh D, Richards M, Hardy R. Cohort profile: the 1946 National Birth Cohort (MRC National Survey of health and development). Int J Epidemiol. 2006;35:49-54

19. Kuh D, Wong A, Shah I, Moore A, Popham M, Curran P, et al. The MRC National Survey of health and development reaches age 70 : maintaining participation at older ages in a birth cohort study. Eur J Epidemiol. 2016;31:1135-47.

20. Alzheimer's Association. Alzheimer's association report: 2014 Alzheimers disease facts and figures. Alzheimers Dement. 2014;10:e47-92.

21. Hardy R, Ghosh AK, Deanfield J, Kuh D, Hughes AD. Birthweight, childhood growth and left ventricular structure at age 60-64 years in a British birth cohort study. Int J Epidemiol. 2016;45(4):1091-102.

22. Medical Research Council, The Wellcome Trust. Framework on the feedback of health-related findings in research 2014. Available from: https://www.mrc. ac.uk/documents/pdf/mrc-wellcome-trust-framework-on-the-feedback-ofhealth-related-findings-in-researchpdf/.

23. Harkins K, Sankar P, Sperling R, Grill JD, Green RC, Johnson KA, et al. Development of a process to disclose amyloid imaging results to cognitively normal older adult research participants. Alzheimers Res Ther. 2015;7:26.

24. Hughes AJ, Daniel SE, Kilford L, Lees AJ. Accuracy of clinical diagnosis of idiopathic Parkinson's disease: a clinico-pathological study of 100 cases. J Neurol Neurosurg Psychiatry. 1992;55:181-4.

25. Spielberger. State-Trait Anxiety Inventory: A comprehensive bibliography. 1983.

26. Eke PI, Dye BA, Wei L, Slade GD, Thornton-Evans GO, Beck JD, et al. Self-reported measures for surveillance of periodontitis. J Dent Res. 2013;92:1041-7.

27. Cerajewska TL, Davies M, West NX. Periodontitis: a potential risk factor for Alzheimer's disease. BDJ Nature Publishing Group. 2015;218:29-34.

28. Briggs $\mathrm{GG}$, Nebes RD. Patterns of hand preference in a student population. Cortex. 1975:11:230-8

29. Bishop DVM. Cerebral asymmetry and language development: cause, correlate, or consequence? Sci Eur PMC Funders. 2013;340:1230531.

30. Postuma RB, Arnulf I, Hogl B, Iranzo A, Miyamoto T, Dauvilliers Y, et al. A single-question screen for rapid eye movement sleep behavior disorder: a multicenter validation study. Mov Disord. 2012;27:913-6.

31. Rami L. Mollica M a, García-Sanchez C, Saldaña J, Sanchez B, Sala I, et al. The subjective cognitive decline questionnaire (SCD-Q): a validation study. J Alzheimers Dis. 2014;41:453-66.

32. Jessen $F$, Amariglio RE, van Boxtel M, Breteler M, Ceccaldi M, Chételat $G$, et al. A conceptual framework for research on subjective cognitive decline in preclinical Alzheimer's disease. Alzheimers Dement. 2014;10:844-52.

33. Galvin JE, Roe CM, Powlishta KK, Coats MA, Muich SJ, Grant E, et al. The AD8: a brief informant interview to detect dementia. Neurology. 2005;65:559-64.

34. Galvin JE, Roe CM, Xiong C, Morris JC. Validity and reliability of the AD8 informant interview in dementia. Neurology. 2006;67:1942-8. 
35. Verghese J, Robbins M, Holtzer R, Zimmerman M, Wang C, Xue X, et al. Gait dysfunction in mild cognitive impairment syndromes. J Am Geriatr Soc Blackwell Publishing Inc. 2008;56:1244-51.

36. Buracchio T, Dodge HH, Howieson D, Wasserman D, Kaye J. The trajectory of gait speed preceding mild cognitive impairment. Arch Neurol. 2010;67:980-6.

37. Hausdorff JM, Yogev G. Cognitive function may be important for fall injury prevention trials. J Am Geriatr Soc. 2006;54:865.

38. Rota V, Perucca L, Simone A, Tesio L. Walk ratio (step length/cadence) as a summary index of neuromotor control of gait: application to multiple sclerosis. Int J Rehabil Res Int Zeitschrift für Rehabil Rev Int Rech réadaptation. 2011;34:265-9.

39. Collett J, Esser P, Khalil H, Busse M, Quinn L, DeBono K, et al. Insights into gait disorders: walking variability using phase plot analysis. Huntington's disease Gait Posture. 2014;40:694-700.

40. Wilson RS, Schneider JA, Bienias JL, Evans DA, Bennett DA. Parkinsonianlike signs and risk of incident Alzheimer disease in older persons. Arch Neurol. 2003;60:539-44.

41. Richards M, Stern Y, Mayeux R. Subtle extrapyramidal signs can predict the development of dementia in elderly individuals. Neurology. 1993;43:2184-8

42. Goetz CG, Tilley BC, Shaftman SR, Stebbins GT, Fahn S, Martinez-Martin P, et al. Movement Disorder Society-sponsored revision of the Unified Parkinson's disease rating scale (MDS-UPDRS): scale presentation and clinimetric testing results. Mov Disord. 2008;23:2129-70.

43. Noyce AJ, Nagy A, Acharya S, Hadavi S, Bestwick JP, Fearnley J, et al. Bradykinesia-akinesia incoordination test: validating an online keyboard test of upper limb function. PLoS One. 2014;9

44. Donohue MC, Sperling RA, Salmon DP, Rentz DM, Raman R, Thomas RG, et al, The preclinical Alzheimer cognitive composite: measuring amyloid-related decline. JAMA Neurol. 2014;71:961-70

45. Hsieh S, Schubert S, Hoon C, Mioshi E, Hodges JR. Validation of the Addenbrooke's Cognitive Examination III in Frontotemporal Dementia and Alzheimer's Disease. Dementia and Geriatric Cognitive Disorders. 2013;36(3-4):242-50.

46. Silverwood RJ, Richards M, Pierce M, Hardy R, Sattar N, Ferro C, et al. Cognitive and kidney function: results from a British birth cohort reaching retirement age. PLoS One. 2014;9:e86743.

47. Folstein MF, Folstein SE, McHugh PR. Mini-mental state. J Psychiatr Res. 1975;12:189-98.

48. Wechsler D. Wechsler memory scale-revised edition. 1987.

49. Wechsler D. Wechsler Adult Intelligence Scale-Revised. 1981.

50. Wechsler D. The Wechsler abbreviated scale of intelligence. San Antonio: The Psychological Corporation; 1999.

51. Shallice T, Stuss DT, Picton TW, Alexander MP, Gillingham S. Multiple effects of prefrontal lesions on task-switching. Front Hum Neurosci. 2007;1:2.

52. Aron AR, Monsell S, Sahakian BJ, Robbins TW. A componential analysis of task-switching deficits associated with lesions of left and right frontal cortex. Brain. 2004;127:1561-73.

53. Hedden $\mathrm{T}$, Oh $\mathrm{H}$, Younger AP, Patel TA. Meta-analysis of amyloid-cognition relations in cognitively normal older adults. Neurology. 2013;80:1341-8.

54. Castel AD, Balota DA, Hutchison KA, Logan JM, Yap MJ. Spatial attention and response control in healthy younger and older adults and individuals with Alzheimer's disease: evidence for disproportionate selection impairments in the Simon task. Neuropsychology. 2007;21:170-82.

55. Hutchison KA, Balota DA, Duchek JM, Ducheck JM. The utility of Stroop task switching as a marker for early-stage Alzheimer's disease. Psychol Aging. 2010;25:545-59.

56. Pertzov Y, Miller TD, Gorgoraptis N, Caine D, Schott JM, Butler C, et al. Binding deficits in memory following medial temporal lobe damage in patients with voltage-gated potassium channel complex antibody-associated limbic encephalitis. Brain. 2013;136:2474-85.

57. Pertzov $Y$, Dong MY, Peich M-C, Husain M. Forgetting what was where: the fragility of object-location binding. PLoS One. 2012;7:e48214.

58. Parra MA, Abrahams S, Logie RH, Méndez LG, Lopera F, Della SS. Visual short-term memory binding deficits in familial Alzheimer's disease. Brain. 2010;133:2702-13.

59. Liang Y, Pertzov Y, Nicholas JM, Henley SMD, Crutch S, Woodward F, et al. Visual short-term memory binding deficit in familial Alzheimer's disease. Cortex. 2016;78:150-64.

60. Pertzov $Y$, Heider M, Liang $Y$, Husain M. Effects of healthy ageing on precision and binding of object location in visual short term memory. Psychol Aging American Psychological Association. 2015;30:26-35.
61. Say MJ, Jones R, Scahill Rl, Dumas EM, Coleman A, Santos RCD, et al. Visuomotor integration deficits precede clinical onset in Huntington's disease. Neuropsychologia Ltd. 2011;49:264-70.

62. Papp KV, Amariglio RE, Dekhtyar M, Roy K, Wigman S, Bamfo R, et al. Development of a psychometrically equivalent short form of the face-name associative memory exam for use along the early Alzheimer's disease trajectory. Clin. Neuropsychol. 2014;28:771-85.

63. Rentz DM, Amariglio RE, Becker JA, Frey M, Olson LE, Frishe K, et al. Face-name associative memory performance is related to amyloid burden in normal elderly. Neuropsychologia. 2011;49:2776-83.

64. Forster S, Lavie N. Failures to ignore entirely irrelevant distractors: the role of load. J Exp Psychol Appl American Psychological Association. 2008;14:73-83.

65. Forster S, Lavie N. Attentional capture by entirely irrelevant distractors. Vis Cogn. 2008;16:200-14.

66. Devanand DP, Lee S, Manly J, Andrews H, Schupf N, Doty RL, et al. Olfactory deficits predict cognitive decline and Alzheimer dementia in an urban community. Neurology. 2015:182-9.

67. Gates GA, Anderson ML, McCurry SM, Feeney MP, Larson EB. Central auditory dysfunction as a harbinger of Alzheimer dementia. Arch Otolaryngol Head Neck Surg. 2011;137:390-5.

68. Lin FR, Albert M. Hearing loss and dementia - who is listening? Aging Ment. Health. 2014;18:671-3.

69. Hardy CJD, Marshall CR, Golden HL, Clark CN, Mummery CJ, Griffiths TD, et al. Hearing and dementia. J Neurol. 2016:1-16.

70. Albers MW, Gilmore GC, Kaye J, Murphy C, Wingfield A, Bennett D a., et al. At the interface of sensory and motor dysfunctions and Alzheimer's disease. Alzheimer's Dement. 2014; 1-29.

71. Bastawrous A, Rono HK, Livingstone IAT, Weiss HA, Jordan S, Kuper H, et al. Development and validation of a Smartphone-based visual acuity test (Peek acuity) for clinical practice and community-based fieldwork. JAMA Ophthalmol. 2015;133:930-7.

72. Doty RL, Shaman P, Dann M. Development of the University of Pennsylvania Smell Identification Test: a standardized microencapsulated test of olfactory function. Physiol Behav. 1984;32:489-502.

73. British Society of Audiology. Recommended Procedure bone-conduction threshold audiometry with and without masking. 2011

74. Rosen S, Souza P, Ekelund C, Majeed A a. Listening to speech in a background of other talkers: effects of talker number and noise vocoding. J Acoust Soc Am 2013;133:2431-43.

75. Clark CM. Schneider J a, Bedell BJ, beach TG, bilker WB, Mintun M a, et al. use of florbetapir-PET for imaging beta-amyloid pathology. JAMA. 2011;305:275-83.

76. Burgos N, Cardoso MJ, Modat M, Punwani S, Atkinson D, Arridge SR, et al. CT synthesis in the head \& amp; neck region for PET/MR attenuation correction: an iterative multi-atlas approach. EJNMMI Phys. 2015 Dec;2(Suppl 1):A31.

77. Burgos N, Cardoso MJ, Thielemans K, Modat M, Pedemonte S, Dickson J, et al. Attenuation correction synthesis for hybrid PET-MR scanners: application to brain studies. IEEE Trans Med Imaging. 2014;33:2332-41.

78. Mugler JP, Brookeman JR. Rapid three-dimensional T1-weighted MR imaging with the MP-RAGE sequence. J Magn Reson Imaging. 1991 Sep-Oct;1(5):561-7.

79. Mugler JP. Optimized three-dimensional fast-spin-echo MRI. J Magn Reson Imaging. 2014;39:745-67.

80. Jovicich J, Czanner S, Greve D, Haley E, van der Kouwe A, Gollub R, et al. Reliability in multi-site structural MRI studies: effects of gradient non-linearity correction on phantom and human data. Neurolmage. 2006;30:436-43.

81. Tustison NJ, Avants BB, Cook PA, Zheng Y, Egan A, Yushkevich PA, et al. N4ITK: improved N3 bias correction. IEEE Trans Med Imaging. 2010;29:1310-20.

82. Cardoso MJ, Modat M, Wolz R, Melbourne A, Cash D, Rueckert D, et al. Geodesic information flows: spatially-variant graphs and their application to segmentation and fusion. IEEE Trans Med Imaging. 2015;34:1976-88.

83. Beckmann CF, DeLuca M, Devlin JT, Smith SM. Investigations into resting-state connectivity using independent component analysis. Philos. Trans R Soc Lond B Biol Sci. 2005;360:1001-13.

84. Mennes M, Jenkinson M, Valabregue R, Buitelaar JK, Beckmann C, Smith S. Optimizing full-brain coverage in human brain MRI through population distributions of brain size. Neurolmage. 2014 Sep;98:513-20.

85. Power JD, Barnes KA, Snyder AZ, Schlaggar BL, Petersen SE. Spurious but systematic correlations in functional connectivity MRI networks arise from subject motion. Neurolmage. 2012;59:2142-54.

86. Modat M, Ridgway GR, Taylor ZA, Lehmann M, Barnes J, Hawkes DJ, et al. Fast free-form deformation using graphics processing units. Comput Methods Prog Biomed. 2010;98:278-84. 
87. Chen G, Saad ZS, Nath AR, Beauchamp MS, Cox RW. FMRI group analysis combining effect estimates and their variances. Neurolmage. 2012;60:747-65.

88. Glover GH, Li TQ, Ress D. Image-based method for retrospective correction of physiological motion effects in fMRI: RETROICOR. Magn Reson Med. 2000;44:162-7.

89. Lohmann G, Stelzer J, Neumann J, Ay N, Turner R. "more is different" in functional magnetic resonance imaging: a review of recent data analysis techniques. Brain Connect. 2013;3:223-39.

90. Hütel M, Melbourne A, Thomas D, Rohrer J and Ourselin S. An overcomplete and efficient ICA for BOLD-fMRI.ISMRM. 2016.

91. Basser PJ, Mattiello J, LeBihan D. MR diffusion tensor spectroscopy and imaging. Biophys J. 1994;66:259-67.

92. Weston PSJ, Simpson IJA, Ryan NS, Ourselin S, Fox NC. Diffusion imaging changes in grey matter in Alzheimer's disease: a potential marker of early neurodegeneration. Alzheimers Res Ther. 2015;7:47.

93. Zhang H, Schneider T. Wheeler-Kingshott C a., Alexander DC. NODDI: practical in vivo neurite orientation dispersion and density imaging of the human brain. Neurolmage. 2012;61:1000-16.

94. Feinberg DA, Jakab PD. Tissue perfusion in humans studied by Fourier velocity distribution, line scan, and echo-planar imaging. Magn Reson Med. 1990;16:280-93.

95. Reese TG, Heid O, Weisskoff RM, Wedeen VJ. Reduction of eddy-current-induced distortion in diffusion MRI using a twice-refocused spin echo. Magn Reson Med. 2003:49:177-82.

96. Andersson JLR, Sotiropoulos SN. An integrated approach to correction for off-resonance effects and subject movement in diffusion MR imaging. Neurolmage. 2016;125:1063-78.

97. Daga P, Pendse T, Modat M, White M, Mancini L, Winston GP, et al. Susceptibility artefact correction using dynamic graph cuts: application to neurosurgery. Med Image Anal. 2014;18:1132-42.

98. Melbourne A, Toussaint N, Owen D, Simpson I, Anthopoulos T,

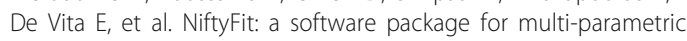
model-fitting of 4D magnetic resonance imaging data. Neuroinformatics. 2016;14:319-37.

99. Cordonnier C, van der Flier WM, Attems J, Bayer A, Bullock R, Jones R, et al. Brain microbleeds and Alzheimer's disease: innocent observation or key player? Brain. 2011;134:335-44.

100. Shoamanesh A, Preis SR, Beiser AS, Vasan RS, Benjamin EJ, Kase CS, et al. Inflammatory biomarkers, cerebral microbleeds, and small vessel disease: Framingham heart study. Neurology. 2015;84:825-32.

101. Haacke EM, Mittal S, Wu Z, Neelavalli J, Cheng Y-CN. Susceptibility-weighted imaging: technical aspects and clinical applications, part 1. AJNR Am J Neuroradiol. 2009;30:19-30.

102. Schweser F, Deistung A, Sommer K, Reichenbach JR. Toward online reconstruction of quantitative susceptibility maps: superfast dipole inversion. Magn Reson Med. 2013;69:1581-93.

103. Vidorreta M, Balteau E, Wang Z, De Vita E, Pastor MA, Thomas DL, et al. Evaluation of segmented 3D acquisition schemes for whole-brain high-resolution arterial spin labeling at 3 T. NMR Biomed. 2014;27:1387-96.

104. Alsop DC, Detre JA, Golay X, Günther M, Hendrikse J, Hernandez-Garcia L, et al. Recommended implementation of arterial spin-labeled perfusion MRI for clinical applications: a consensus of the ISMRM perfusion study group and the European consortium for ASL in dementia. Magn Reson Med. 2015;73:102-16.

105. Leung KK, Barnes J, Modat M, Ridgway GR, Bartlett JW, Fox NC, et al. Brain MAPS: an automated, accurate and robust brain extraction technique using a template library. Neurolmage. 2011;55:1091-108.

106. Jorge Cardoso M, Leung K, Modat M, Keihaninejad S, Cash D, Barnes J, et al. STEPS: similarity and truth estimation for propagated segmentations and its application to hippocampal segmentation and brain parcelation. Med Image Anal. 2013;17:671-84.

107. Malone IB, Leung KK, Clegg S, Barnes J, Whitwell JL, Ashburner J, et al. Accurate automatic estimation of total intracranial volume: a nuisance variable with less nuisance. Neurolmage. 2015;104:366-72.

108. Clarkson MJ, Cardoso MJ, Ridgway GR, Modat M, Leung KK, Rohrer JD, et al. A comparison of voxel and surface based cortical thickness estimation methods. Neurolmage. 2011;57:856-65.

109. Gregoire SM, Chaudhary UJ, Brown MM, Yousry TA, Kallis C, Jäger HR, et al. The Microbleed anatomical rating scale (MARS): reliability of a tool to map brain microbleeds. Neurology. 2009;73:1759-66.
110. Wahlund LO, Barkhof F, Fazekas F, Bronge L, Augustin M, Sjögren M, et al. A new rating scale for age-related white matter changes applicable to MRI and CT. Stroke. 2001;32:1318-22.

111. Prins ND, van Straaten ECW, van Dijk EJ, Simoni M, van Schijndel RA, Vrooman $\mathrm{HA}$, et al. Measuring progression of cerebral white matter lesions on MRI: visual rating and volumetrics. Neurology. 2004;62:1533-9.

112. Jain S, Sima DM, Ribbens A, Cambron M, Maertens A, Van Hecke W, et al. Automatic segmentation and volumetry of multiple sclerosis brain lesions from MR images. Neurolmage Clin. 2015;8:367-75.

113. Leung KK, Clarkson MJ, Bartlett JW, Clegg S, Jack CR, Weiner MW, et al. Robust atrophy rate measurement in Alzheimer's disease using multi-site serial MRI: tissue-specific intensity normalization and parameter selection. Neurolmage. 2010;50:516-23.

114. Yang L, Rieves D, Ganley C. Brain Amyloid Imaging — FDA Approval of Florbetapir F18 Injection. http://dx.doi.org/10.1056/NEJMp1208061. Massachusetts Medical. Society; 2012;

115. Andrews KA, Modat M, Macdonald KE, Yeatman T, Cardoso MJ, Leung KK, et al. Atrophy rates in asymptomatic Amyloidosis: implications for Alzheimer prevention trials. PLoS One. 2013;8

116. Erlandsson K, Buvat I, Pretorius PH, Thomas BA, Hutton BF. A review of partial volume correction techniques for emission tomography and their applications in neurology, cardiology and oncology. Phys Med Biol. 2012;57:R119-59.

117. O'Bryant SE, Gupta V, Henriksen K, Edwards M, Jeromin A, Lista S, et al. Guidelines for the standardization of preanalytic variables for blood-based biomarker studies in Alzheimer's disease research. Alzheimers Dement. 2015;11:549-60.

118. Rohrer JD, Woollacott IOC, Dick KM, Brotherhood E, Gordon E, Fellows A, et al. Serum neurofilament light chain protein is a measure of disease intensity in frontotemporal dementia. Neurology. 2016 Sep;27:87(13).

119. Mattsson N, Zetterberg $H$, Janelidze S, Insel PS, Andreasson U, Stomrud E, et al. Plasma tau in Alzheimer disease. Neurology. 2016;87:1827-35.

120. Janelidze S, Stomrud E, Palmqvist S, Zetterberg $H$, van Westen D, Jeromin A, et al. Plasma $\beta$-amyloid in Alzheimer's disease and vascular disease. Sci Rep. 2016;6:26801.

121. Gisslén M, Price RW, Andreasson U, Norgren N, Nilsson S, Hagberg L, et al. Plasma concentration of the Neurofilament light protein (NFL) is a biomarker of CNS injury in HIV infection: a cross-sectional study. EBioMedicine. 2016;3:135-40

122. Shah T, Engmann J, Dale C, Shah S, White J, Giambartolomei C, et al. Population genomics of Cardiometabolic traits: Design of the University College London-London School of Hygiene and Tropical MedicineEdinburgh-Bristol (UCLEB) consortium. PLoS One. 2013;8:e71345.

123. Nalls MA, Bras J, Hernandez DG, Keller MF, Majounie E, Renton AE, et al. NeuroX, a fast and efficient genotyping platform for investigation of neurodegenerative diseases. Neurobiol Aging. 2015;36:1605.e7-12.

124. Gaysina D, Gardner MP, Richards M, Ben-Shlomo Y. Cortisol and cognitive function in midlife: the role of childhood cognition and educational attainment. Psychoneuroendocrinology. 2014;47:189-98.

125. Ross JM, Simpson HR. The National Survey of health and development: 1. Educational Attainment Br J Educ Psychol. 1971;41:49-61.

126. Ross JM, Simpson HR. The National Survey of health and development: 2 . Rate of school progress between 8 and 15 years and between 15 and 18 years. Br J Educ Psychol 1971; 41:125-135.

127. Gale CR, Cooper R, Craig L, Elliott J, Kuh D, Richards M, et al. Cognitive function in childhood and lifetime cognitive change in relation to mental wellbeing in four cohorts of older people. PLoS One. 2012;7:e44860.

128. Stewart R, Hardy R, Richards M. Associations between skeletal growth in childhood and cognitive function in mid-life in a 53-year prospective birth cohort study. PLoS One. 2015;10:e0124163.

129. Albanese E, Hardy R, Wills A, Kuh D, Guralnik J, Richards M. No association between gain in body mass index across the life course and midlife cognitive function and cognitive reserve-the 1946 British birth cohort study. Alzheimers Dement. 2012;8:470-82.

130. Richards M, Hardy R, Wadsworth M. Alcohol consumption and midlife cognitive change in the British 1946 birth cohort study. Alcohol Alcohol. 2004:40:112-7.

131. Richards M, Strachan D, Hardy R, Kuh D, Wadsworth M. Lung function and cognitive ability in a longitudinal birth cohort study. Psychosom Med. 2005;67:602-8. 
132. Richards M, Barnett JH, Xu MK, Croudace TJ, Gaysina D, Kuh D, et al. Lifetime affect and midlife cognitive function: prospective birth cohort study. Br J Psychiatry. 2014;204:194-9.

133. Hurst L, Stafford M, Cooper R, Hardy R, Richards M, Kuh D. Lifetime socioeconomic inequalities in physical and cognitive aging. Am J Public Health. 2013; 103:1641-8

Submit your next manuscript to BioMed Central and we will help you at every step:

- We accept pre-submission inquiries

- Our selector tool helps you to find the most relevant journal

- We provide round the clock customer support

- Convenient online submission

- Thorough peer review

- Inclusion in PubMed and all major indexing services

- Maximum visibility for your research

Submit your manuscript at www.biomedcentral.com/submit 